\title{
L'anorexie mentale chez les adolescents et les jeunes adultes de sexe masculin : Recension des écrits
}

\author{
par \\ Laurence Corbeil-Serre \\ Département de Psychologie \\ Faculté des arts et des sciences
}

Essai doctoral présenté à la Faculté des arts et des sciences en vue de l'obtention du grade de Doctorat en Psychologie option D.Psy

Août, 2012

(C) Laurence Corbeil-Serre, 2012 


\section{Résumé}

L'anorexie mentale a été la cible de beaucoup d'efforts en recherche. Ces derniers ont cependant été principalement concentrés sur l'étude de femmes. Les écrits sur les garçons atteints d'anorexie mentale étant actuellement émergents, la présente recension des écrits propose une mise en commun des connaissances concernant cette population pouvant être dégagées des recherches publiées entre 1994 et 2011. Les variables spécifiquement ciblées sont la présentation clinique, les conditions préalables au trouble, les caractéristiques de la personnalité, l'orientation et l'activité sexuelles, l'identité de genre, les troubles comorbides et le pronostic. La mise en commun et les comparaisons des résultats des 24 études constituant la recension nous permettent d'appuyer des constats émis par des recherches antérieures et de dégager certaines tendances, dont celle qu'il semble exister des similarités entre la symptomatologie comportementale des garçons et des filles. La recension permet de dégager certains aspects où des recherches supplémentaires sont nécessaires et de souligner la pluralité et l'hétérogénéité des méthodes de recherche.

Mots-clés: Anorexie, garçons, tableau clinique, conditions préalables, personnalité, orientation sexuelle, activité sexuelle, identité de genre, troubles comorbides, pronostic 
Abstract: Anorexia nervosa has been the target of many research efforts. The latest studies, however, are mainly focused on female subjects. The literature on males with anorexia nervosa is currently emerging. The review of this literature suggests pooling knowledge about male anorexia nervosa that can be extracted from the literature starting from1996 until 2011. The variables that are specifically targeted are clinical presentation, the premorbid conditions, personality characteristics, sexual orientation and gender identity, comorbid troubles, and prognosis. The comparison and the pooling of the results of 24 studies forming the review allow us in some cases to support observations made by previous research and to identify certain trends. Among other things, there appears to be similarities between the behavioral symptoms of boys and girls. The review identifies some areas where further research is needed and emphasizes the plurality and heterogeneity of research methods.

Keywords : anorexia, males, clinical presentation, premorbid conditions, personality, sexual orientation, sexual activity, gender identity, comorbidity, prognosis 


\section{Table des matières}

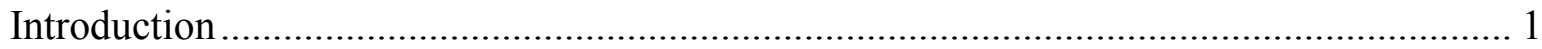

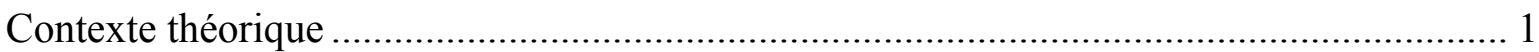

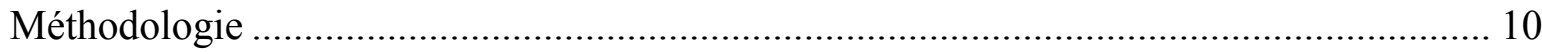

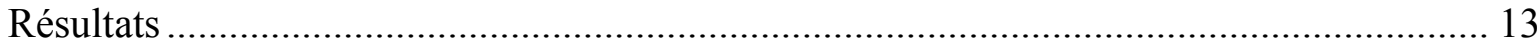

Descriptions des participants et méthodologies .................................................... 14

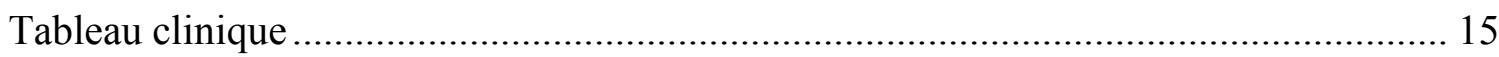

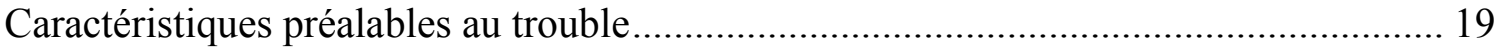

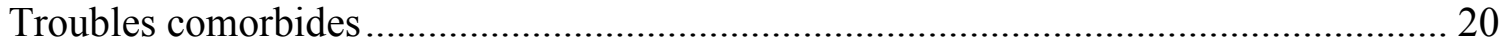

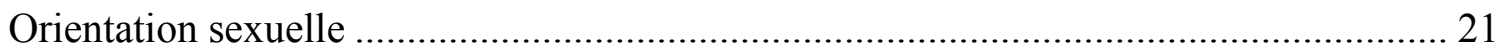

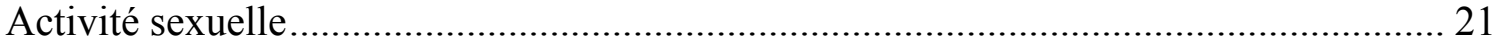

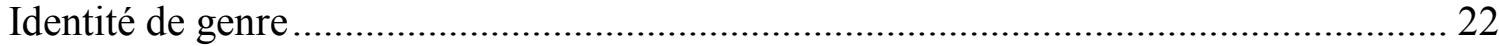

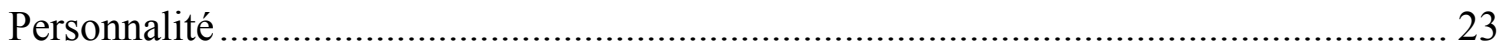

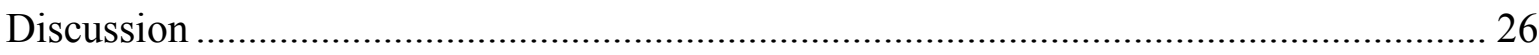

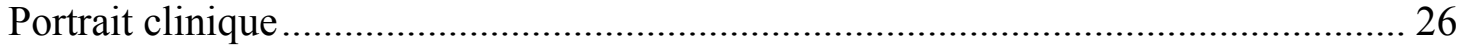

Caractéristiques psychologiques de l'anorexie chez les garçons ............................. 33

Orientation sexuelle et identité de genre .............................................................. 33

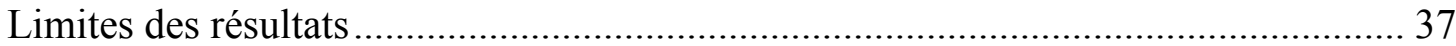

Piste et suggestion pour la recherche future ...................................................... 39

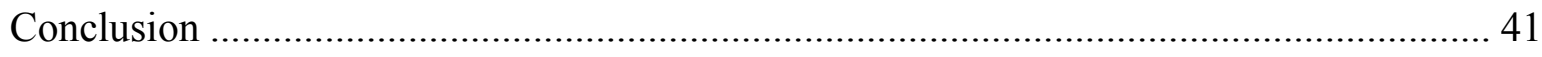

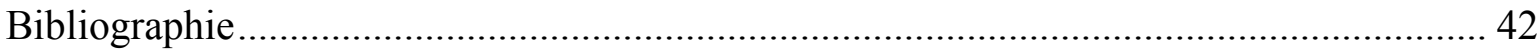

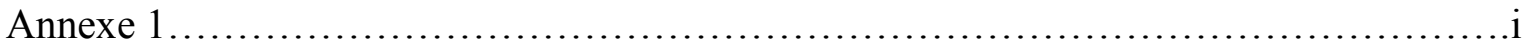




\section{Introduction}

Des troubles de la conduite alimentaire (TCA), c'est l'anorexie mentale (AN) qui a suscité le plus d'intérêt dans les écrits scientifiques, les premières descriptions datant de la fin du 19e siècle (Chambry \& Agman, 2006; Striegel-Moore \& Bulik, 2007). Plusieurs des connaissances acquises à ce jour sont cependant tirées de l'étude de femmes présentant ce trouble (Chambry, 2002). Dans le passé, des études sur les garçons atteints de trouble de la conduite alimentaire (TCA), dont l'AN, ont été effectuées, mais c'est depuis les années 1980 que leur qualité méthodologique s'est améliorée, entre autres en ce qui concerne la taille des échantillons (Burns, 1985; Fernandez-Aranda et al., 2004). La connaissance de l'AN chez les garçons reste toutefois limitée et les résultats de recherches divergent sur plusieurs aspects. L'objectif de cet essai est de faire une recension des écrits portant sur les garçons adolescents et les jeunes adultes qui présentent un problème d'AN dans le but de faire le point sur les connaissances actuelles et de déterminer les aspects pour lesquels des recherches additionnelles seraient nécessaires.

\section{Contexte théorique}

Les critères diagnostiques de l'anorexie mentale sont les suivants: un refus de maintenir un poids corporel d'une valeur minimale normale se concrétisant par une diminution du poids en deçà de $85 \%$ du poids attendu pour la taille et l'âge; une peur intense de prendre du poids; une altération significative de la manière de percevoir son corps et un «déni de la gravité de la maigreur actuelle » malgré les apparentes pertes de poids et leurs conséquences. Le dernier critère diagnostique ne s'applique qu'aux femmes 
post pubères; il s'agit d'une aménorrhée présente depuis trois cycles menstruels (APA, 2004). Pour recevoir un diagnostic d'AN, les symptômes ne doivent pas être attribuables à une autre problématique physique ou psychiatrique (Freeman, 2005; Reijonen, 2003; Robert-McComb, 2001).

On distingue deux types d'AN. Dans le type restrictif (AN-R), les comportements de pertes de poids se traduisent essentiellement par la restriction alimentaire et l'exercice physique. Le type avec crises de boulimie, vomissements et prise de purgatifs (AN-B), est caractérisé par la présence régulière de crises de boulimie (ingestion en moins de deux heures d'une plus grande quantité de nourriture que ce qui est attendu accompagnée d'un sentiment de perte contrôle) ou par le recours aux vomissements provoqués ou par la prise de laxatifs (APA, 2004). L'évolution de la maladie peut entraîner un mouvement d'un type vers l'autre, le plus souvent dans le sens d'une AN-R vers une AN-B (Linschied, 2003; Robert-McComb, 2001). La proportion de la population générale atteinte d'AN est estimée entre $0,5 \%$ et $3 \%$ (Becker, 1999; Haas, 2001; Linschied, 2003; Walcott, 2003). Ce trouble se développe typiquement durant l'adolescence et atteint son apogée entre l'âge de 14 ans et de 18 ans (Becker, 1999; Comerci, 1989; Haas, 2001; Linschied, 2003; Ray, 2004; Robert-McComb, 2001; Striegel-Moore \& Bulik, 2007). L'étiologie exacte de l'AN n'est pas clairement déterminée, mais il est généralement admis par les experts qu'elle serait issue d'une combinaison de facteurs (Garfinkel \& Garner, 1982; Linschied, 2003; Reijonen, 2003; Striegel-Moore, 1997). Ces derniers peuvent être regroupés en plusieurs catégories : les facteurs génétiques, physiologiques, développementaux, socioculturels, familiaux, 
individuels, comportementaux et cognitifs (Aime, 2008; Domine, 2009; Halmi et al., 2000; Linschied, 2003; Pratt, 2003; Striegel-Moore, 1997).

Selon Becker (1999), les adolescents présentant des symptômes d'AN risquent plus de souffrir d'autres problématiques psychologiques ou médicales, particulièrement ceux souffrant d'AN-B (Pratt, 2003). Il est cependant impossible d'affirmer si l'AN favorise le développement d'autres psychopathologies ou si c'est plutôt l'inverse (Pratt, 2003). Les troubles ou les symptômes des troubles suivants sont fréquemment associés à l'AN: les troubles dépressifs, anxieux, obsessionnels-compulsifs, de l'humeur et certains troubles de la personnalité (APA, 2003, 1993; Becker, 1999; Bryant-Waugh, 2007; Lock, 2007; Pratt, 2003; Reijonen, 2003). Les personnes souffrant d'AN-B sont aussi plus fréquemment sujettes à d'autres troubles liés au contrôle des impulsions (ex : abus de substances, tentatives de suicide) et ont plus souvent des caractéristiques de la personnalité limite que celles atteintes d'AN-R (APA, 2004).

L’AN est associée à un taux de mortalité élevé (5-15\%) (Andersen, 1990; Comerci, 1989; Herzog, 2000; Lock, Reisel, \& Steiner, 2001; Reijonen, 2003; Romeo, 1994) qui augmente avec la durée du follow-up passant d'environ $5 \%$ entre quatre et dix ans de suivi à approximativement $12 \%$ lorsque le suivi dépasse 10 ans (Steinhausen, 2002). Certaines personnes répondent tout de même bien aux interventions proposées (Becker, 1999; Comerci, 1989; Haas, 2001). Pour les femmes adultes, on observe des taux de rémission complète pour $30 \%$ à $50 \%$ d'entre elles et des taux d'amélioration partielle de la condition avec maintien de certains symptômes chez 30 \% à 40 \% (Becker, 1999; Comerci, 1989; Haas, 2001). Cependant, chez $20 \%$ à $30 \%$ d'entre elles, le traitement n'entraîne pas 
d'amélioration et le trouble est alors qualifié de chronique (Becker, 1999; Comerci, 1989; Haas, 2001). Chez les adolescents, le pronostic est plus favorable. Dans une méta-analyse portant sur 119 études, Steinhausen (2002) rapporte des taux de 57,1\% de rémission complète, de $25,9 \%$ d'amélioration et de $16,9 \%$ de chronicité pour les adolescents. Selon Comerci (1989), l'obésité prémorbide, des pertes de poids extrêmes, un début tardif du trouble et la présence de purges comptent parmi les prédicteurs d'un pronostic plus sombre.

Prévalence de l'anorexie chez les garçons. Il est généralement estimé qu'entre $5 \%$ à $15 \%$ des populations cliniques atteintes de TCA sont de sexe masculin (Becker, 1999; Freeman, 2005; Gadalla, 2008; Muise, 2003; Oyebode, 1988; Ray, 2004; Reijonen, 2003; Romeo, 1994; Weltzin, 2005). Pour l'AN, le ratio est d'environ un homme pour dix femmes chez les adultes (APA, 2004; Comerci, 1989). Chez les adolescents, les chiffres sont plus variables. Muise (2003) rapporte un ratio d'un garçon pour 2,33 filles dans une étude effectuée auprès de populations d'adolescents prépubères, tandis qu'il serait d'un garçon pour 19 filles chez les adolescents postpubères. Plusieurs sont d'avis que les chiffres disponibles sous-estimeraient le pourcentage réel d'individus de sexe masculin atteints d'anorexie (Burns, 1984; Chambry \& Agman, 2006; Romeo, 1994).

Certains obstacles compliquent en effet la détection de l'AN chez les hommes (Walcott, 2003). D’abord, les critères diagnostiques de l'AN et les instruments de dépistage sont élaborés autour de préoccupations corporelles et pondérales caractéristiques des femmes, soit la recherche de la minceur et la présence de comportements associés aux régimes alimentaires (Chambry, 2002). Certains chercheurs émettent l'hypothèse qu'ils ne cerneraient peut-être pas les symptômes tels qu'ils se présentent chez les garçons dont les 
préoccupations seraient plutôt centrées sur la masse musculaire (Fernandez-Aranda, et al., 2004; Lock, et al., 2001). Ensuite, il n'existe actuellement pas de marqueur physique dans les critères diagnostiques de l'AN chez les garçons (Crosscope-Happel, 2000; Freeman, 2005; Muise, 2003). Par ailleurs, certains chercheurs se penchent actuellement sur la possibilité d'inclure la diminution de la testostérone comme équivalent de l'aménorrhée (Andersen, 1982 cité dans Becker, 1999). Un autre obstacle au diagnostic serait une moins grande tendance des cliniciens à suspecter l'AN chez les hommes, entre autres à cause des connaissances limitées du trouble chez cette population. Enfin, la honte vécue par plusieurs jeunes hommes face à leur problème, plus connu chez les populations féminines, peut les mener à le dissimuler et les rendre plus réticents à chercher de l'aide (Andersen, 1990; Carlat, 1997; Domine, 2009; Harvey, 2003; Olivardia, 1995; Ray, 2004). Malgré ces divers obstacles, la problématique de l'anorexie masculine est actuellement reconnue et, bien qu'elles soient encore peu développées, certaines connaissances spécifiques à ce groupe commencent à émerger des écrits scientifiques.

Caractéristiques comportementales et descriptives de l'anorexie chez les garçons. Bien que les résultats de recherche soient très hétérogènes, les écrits scientifiques relèvent certaines similitudes entre la symptomatologie de l'AN chez les garçons et chez les filles (Aime, 2008; Burns, 1985; Chambry \& Agman, 2006; Chambry, 2002; Fichter, 1985; Olivardia, Pope, Mangweth, \& Hudson, 1995; Woodside, Garfinkel, Lin, Goering, \& Kaplan, 2001). Comme les filles anorexiques, les garçons auraient peur de perdre le contrôle de leur poids (Chambry, 2002) et de devenir gros (Fichter, 1985; Herzog, 1984; Romeo, 1994), ils auraient un idéal corporel de minceur (Fichter, 1985), des distorsions dans la manière de 
percevoir leur corps (Chambry, 2002) et nieraient leur trouble (Fichter, 1985; Romeo, 1994). Comme elles, ils auraient recours aux restrictions alimentaires et au jeûne et, plus ou moins fréquemment selon les études, aux vomissements provoqués et à l'usage de laxatifs comme méthodes de contrôle de leur poids (Chambry, 2002; Fichter, 1985; Weltzin, 2005). Les garçons seraient aussi sujets aux crises de boulimie (Chambry, 2002).

Toutefois, des auteurs avancent que les présentations cliniques des garçons différeraient sur certains aspects. L'anorexie restrictive serait plus rare (Chambry, 2002), on observerait plus d'hyperactivité physique comme méthode de contrôle du poids (Fichter, 1985; Weltzin, 2005) et leurs préoccupations se centreraient plus autour de la masse musculaire que sur le poids ( Andersen, 1999; Bryant-Waugh, 2007; Harvey, 2003; Walcott, 2003; Weltzin, 2005).

Outre les aspects de la symptomatologie, d'autres comparaisons ont été effectuées entre les sexes et ont généré des résultats souvent divergents. Un élément fréquemment abordé dans les écrits est l'âge d'apparition du trouble. Plusieurs auteurs avancent que, comme chez les filles, l'AN se manifesterait généralement chez les garçons entre 13 ans et 19 ans (Chambry, 2002; Fichter, 1985). Par contre, d'autres chercheurs observent une apparition plus tardive chez ces derniers, soit vers la fin de l'adolescence et au début de l'âge adulte (Herzog, 1984; Walcott, 2003).

Un autre élément de comparaison entre les sexes est le poids avant l'apparition de l'AN. Certains résultats de recherche soutiennent qu'à l'apparition des symptômes, les garçons auraient un poids plus élevé que les filles et qu'une plus grande proportion d'entre eux aurait un poids supérieur au poids attendu avant le début du trouble (Chambry, 2002). 
En contrepartie, certains observent des proportions similaires d'obésité prémorbide chez les deux sexes (Herzog, 1984).

En ce qui a trait à la personnalité, certains auteurs émettent l'hypothèse qu'il y aurait des facteurs de protection inhérents à la personnalité des hommes, comme un perfectionnisme moindre, qui diminueraient leur risque de développer un TCA (Woodside et al., 2004). D'autres auteurs ne retrouvent cependant pas cette différence quant au perfectionnisme (Fernandez-Aranda, et al., 2004). Lorsque les hommes et les femmes anorexiques sont comparés sur d'autres aspects de la personnalité, les scores des hommes sont plus bas pour la dépendance aux renforcements, pour l'esprit de coopération, pour l'évitement du danger et pour la préoccupation pour les erreurs (Woodside, et al., 2004). Certains suggèrent aussi que, chez les hommes, l'AN serait plus fréquemment associée à une structure de personnalité psychotique (Bercé, 2009).

Dans certaines études comparant les garçons et les filles, on relève une différence importante dans le domaine de la sexualité. Selon certains auteurs, avant le début de leur trouble, les garçons auraient vécu moins d'expériences sexuelles réelles ou fantasmatiques et éprouveraient plus d'anxiété par rapport à la sexualité (Chambry \& Agman, 2006; Herzog, 1984). Certains soutiennent aussi la présence d'une plus grande insatisfaction liée à l'identité sexuelle (Chambry \& Agman, 2006) et plus de confusion au niveau de l'identité de genre (Fichter, 1985; Romeo, 1994) chez les garçons.

Toujours en ce qui a trait à la sexualité, des auteurs avancent que le taux d'homosexualité serait considérablement plus élevé chez les garçons (Herzog, 1984). Les résultats des études concernant la prévalence de l'homosexualité sont très variables et 
oscillent entre $20 \%$ et $58 \%$ (Andersen, 1999; Carlat, 1997; Chambry, 2002; Herzog, 1984). Si l'on considère le pourcentage le plus faible d'homosexualité rapporté dans les études citées ci-haut, soit le $20 \%$ obtenu dans l'étude d'Andersen (1999), celui-ci reste tout de même quatre à cinq fois plus élevé que les pourcentages retrouvés dans la population générale (3 à $5 \%$ ) et chez les femmes anorexiques (4 \%) (Herzog, 1984). Deux hypothèses sont fréquemment évoquées pour expliquer l'importance de la présence de l'homosexualité chez les hommes anorexiques. La première est que les hommes homosexuels auraient plus tendance à demander de l'aide pour leur trouble, l'AN serait donc mieux dépistée chez eux que chez les hommes hétérosexuels (Chambry, 2002). La seconde hypothèse, évoquée par plusieurs auteurs, voudrait que l'importance de l'idéal de minceur dans les communautés homosexuelles masculines augmenterait les risques de souffrir d'AN (Andersen, 1990; Chambry, 2002; Herzog, 1984; Striegel-Moore \& Bulik, 2007).

Un dernier élément souvent assujetti à la comparaison entre les garçons et les filles est le pronostic. En effet, certains auteurs soutiennent que les garçons souffrants d'AN auraient un pourcentage de rémission complète inférieur à celui des filles (Harvey, 2003; Oyebode, 1988; Romeo, 1994; Walcott, 2003), tandis que d'autres observent à l'inverse que même si les hommes rencontrent plus d'obstacles durant leur traitement, ils auraient un pronostic comparable à celui des femmes (Andersen, 1999; Chambry, 2002; Fichter, 1985; Weltzin, 2005).

Certains prédicteurs du pronostic de l'AN seraient communs aux deux sexes (Burns, 1985). Les principaux facteurs liés à un mauvais pronostic fréquemment évoqués dans les écrits scientifiques sont les suivants: un âge plus élevé au début de la maladie (Steinhausen, 
2002) et à la prise en charge (Chambry, 2002), un traitement antérieur pour un épisode d'AN et des pertes de poids importantes (Burns, 1985), une longue durée des symptômes avant la prise en charge (Chambry, 2002; Steinhausen, 2002), le retrait social (Chambry, 2002), les relations familiales complexes et dans lesquelles l'expression des affects n'est pas encouragée (Burns, 1985; Chambry, 2002) et la présence de vomissements, d'abus de purgatifs et de crises de boulimies (Steinhausen, 2002). En ce qui concerne les prédicteurs d'un pronostic favorable, on trouve les bonnes relations parents enfants (Steinhausen, 2002; Weltzin, 2005) et la capacité de la famille à s'engager dans une thérapie familiale (Chambry, 2002), un diagnostic précoce, une intervention rapide, la présence de soutien social, la prise de poids durant le traitement et une bonne estime de soi (Weltzin, 2005). Plusieurs auteurs suggèrent que la présence d'activité sexuelle réelle ou fantasmatique antérieure à l'apparition du trouble serait un facteur de bon pronostic pour les garçons (Andersen, 1999; Burns, 1985; Chambry, 2002).

Comme il est possible de le constater, les connaissances émergentes des écrits sur l'AN masculine sont issues de résultats de recherche divergents. Elles gagneraient donc à être dégagées des recherches actuelles pour permettre de se prononcer sur leurs caractéristiques spécifiques et de relever les dimensions qui sont encore mal connues. Cette intégration des connaissances serait pertinente tant pour favoriser une meilleure reconnaissance du trouble par les intervenants que pour orienter la recherche vers les aspects les moins bien connus de l'AN masculine. 


\section{Méthodologie}

L'intérêt de cet essai est de tenter de regrouper les diverses informations concernant les caractéristiques de la présentation clinique et les aspects psychologiques de l'AN chez les garçons. Plusieurs des études effectuées jusqu'à présent auprès des garçons atteints d'AN portent sur un ou quelques aspects spécifiques de cette problématique, si bien qu'il est difficile pour un lecteur n'étant pas expert dans le domaine ou s'y intéressant nouvellement de se faire une idée de ce que l'on sait ou pas de l'AN masculine. L'objectif est donc d'effectuer une recension des écrits scientifiques portant sur les adolescents et les jeunes adultes atteints d'AN dans le but de dégager l'état des connaissances actuelles sur l'AN masculine. La recension porte sur neuf variables identifiées comme pertinentes en raison soit de leur récurrence dans les écrits scientifiques ou soit des résultats divergents repérés dans les recherches. Ces dernières sont la présentation du tableau clinique, les caractéristiques préalables au trouble, les caractéristiques de la personnalité, l'activité sexuelle, l'orientation sexuelle, l'identité de genre, les comorbidités psychiatriques et le pronostic. La méthodologie a aussi été ajoutée comme variable d'intérêt dans le but de porter un regard critique sur les résultats des recherches incluses dans la recension. Les articles retenus sont ceux présentant toutes formes de recherches soit les études empiriques avec ou sans groupe contrôle, les études de cas et les observations cliniques. Les articles théoriques ont été exclus.

Recension des articles. La sélection des articles s'est faite à partir de la base de données informatisée PsycINFO et a été effectuée de manière plus large que restrictive afin 
d'identifier un nombre maximal d'articles. La base de données PsycINFO a été sélectionnée en raison de l'aspect plus psychologique que médical des variables ciblées par la recension. Les études recherchées sont celles publiées entre 1994 et 2011, rédigées en anglais ou en français. Le choix d'inclure une restriction temporelle repose sur l'intention de faire une analyse en profondeur de chacun des articles inclus dans la recension ainsi que sur l'objectif de faire le point sur les connaissances récemment développées sur l'AN masculine. L'année 1994 a été déterminée comme limite en lien avec la parution du DSMIV et dans le but de favoriser une homogénéité des critères utilisés pour le diagnostic des participants dans les études.

Descripteurs. Lors des recherches dans les bases de données, les troncatures et les codes d'index ont été utilisés pour assurer la reconnaissance des pluriels des descripteurs et pour mieux cibler l'investigation. Après l'essai de différentes combinaisons, les descripteurs suivants ont été utilisés : Anorexia, Anorec*, Eating Disorders, Boys et Males; ils ont généré 414 articles dans PsycINFO. De ces 414 articles, 390 étaient consacrés à des groupes issus de la population générale ou concernaient des formes sous-cliniques de l'AN ou d'autres TCA: ils ont été exclus afin d'avoir un échantillon plus homogène. Les recherches dans les bases de données ont permis de constater qu'un nombre limité d'études (11) portaient exclusivement sur les adolescents de sexe masculin. Dans le but de maximiser l'information sur cette population, les articles dont les groupes comprenaient des adolescents et de jeunes adultes ont été inclus. 
Critères de sélection des articles. Les études retenues satisfont les critères d'inclusion suivants : elles ont été publiées entre 1994 et 2011, elles traitent de populations cliniques masculines (celles incluant des filles ont été retenues si elles présentaient au moins une partie de leurs résultats de manière distincte pour les deux groupes), elles comportent des groupes composés d'adolescents ou de jeunes adultes et elles ciblent des groupes ayant reçu un diagnostic d'AN (lorsque l'article comprend aussi des participants présentant d'autres TCA, il est retenu s'il présente des résultats séparés pour les différents groupes diagnostiques). Les études qui ont été mises de côté (critères d'exclusion) sont celles qui ont été publiées avant 1994, celles qui traitent de populations féminines ou qui présentent seulement des résultats communs pour les deux sexes, celles qui ont pour participants des personnes présentant un autre TCA ou avec des résultats communs pour les différents groupes diagnostiques, ainsi que celles effectuées auprès de populations non cliniques. À la suite des recherches dans les bases de données, le processus de sélection des articles a débuté par la lecture des résumés des articles identifiés par les recherches ainsi que par une vérification de la réelle correspondance aux critères d'inclusion. Au terme du processus, 24 articles ont été retenus. Ils sont identifiés par un astérisque dans la liste de référence.

Analyse des articles. Les articles sélectionnés ont été analysés à l'aide d'une grille ayant été élaborée sur la base des neuf variables d'intérêts : la méthodologie des études, le tableau clinique, les conditions préalables au trouble, les caractéristiques de la personnalité, l'orientation sexuelle, l'activité sexuelle, l'identité de genre, les troubles comorbides et le pronostic. Les variables d'intérêt ont été sélectionnées en raison de l'importance de leur 
présence dans les écrits scientifiques ainsi que de la variabilité des conclusions des études les ayant abordées. Les informations sur les variables ciblées ont ainsi été extraites et répertoriées dans la grille pour ensuite être mises en commun dans le but de dégager des tendances, des consensus et des divergences dans les résultats pour chacune d'entre elles. Les méthodologies des études ont été considérées dans l'interprétation des résultats. L'ensemble des variables n'étant pas abordé dans tous les articles, nous nous sommes retrouvés avec plusieurs données manquantes. Dans le but de pallier à cette réalité, nous avons décidé de présenter les résultats en indiquant le pourcentage de fréquence basé uniquement sur le nombre de participants pour lesquels des informations étaient disponibles. Ainsi le lecteur pourra constater que les pourcentages ne totalisent pas toujours $100 \%$.

\section{Résultats}

Cette section présente les principaux résultats de la recension. Ceux-ci ont été regroupés en neuf sections correspondantes aux variables à l'étude. Au début de chacune, il est précisé pour quel pourcentage de l'échantillon total l'information est disponible pour cette variable donnée. Les pourcentages subséquents sont issus de ce premier pourcentage de l'échantillon total et portent uniquement sur ce dernier. En raison de la diversité des variables étudiées, de la grande variété des méthodologies utilisées, ainsi que du nombre parfois très restreint de participants pour lesquels de l'information était disponible dans les études, une pondération des résultats en fonction de la qualité méthodologique des études s'est avérée impossible. 


\section{Descriptions des participants et méthodologies}

La recension comprend 24 articles qui regroupent un total de 279 participants de sexe masculin âgés entre 11 ans et 36 ans. L'âge moyen des participants aux études est estimé à 18,4 ans avec un écart type de 4,38. Onze études sont constituées de participants exclusivement adolescents (moins de 18 ans), six études s'intéressent exclusivement aux adultes (18 à 36 ans) et sept ont des échantillons mixtes c'est-à-dire composés d'adolescents et de jeunes adultes. La presque totalité des participants aux études $(99,3 \%)$ a été recrutée durant ou après leur passage dans des unités de traitement en milieu hospitalier. Dix articles mentionnent les critères ayant été utilisés pour le diagnostic de l'anorexie mentale. Dans neuf de ses études, les critères étaient ceux du DSM et dans deux autres, ceux de la CIM (en plus de ceux du DSM pour l'une d'elles).

De l'ensemble des articles, 13 (54,2\% des études) sont des études de cas ayant entre un et cinq participants $(\mathrm{N}$ total $=19)$. Neuf articles $(37,5 \%$ des études $)$ présentent des études descriptives dont la taille de l'échantillon varie entre sept et 62 participants $(\mathrm{N}$ total= 213) : parmi celles-ci, six ont un groupe de comparaison. Les deux dernières sont des études rétrospectives ( $8,3 \%$ des études), l'une a un groupe de comparaison et leur nombre total de participants est de 46 .

Pour répondre à leurs objectifs, onze études $(45,8 \%$ des études, $\mathrm{N}=154)$ ont utilisé des instruments psychométriques. Dans l'ensemble, on rapporte avoir utilisé douze questionnaires, cinq entrevues semi-structurées, un test projectif, deux tests comparant la silhouette réelle à la silhouette perçue ainsi qu'un test évaluant la personnalité sur la base de performance motrice. Environ les deux tiers des questionnaires utilisés ont fait leurs 
preuves au plan de leurs qualités psychométriques, celles-ci étant soit rapportées par les auteurs ou accessibles dans les écrits scientifiques. En ce qui concerne les autres outils de mesure (entrevues semi-structurées, tests projectifs, tests comparant la silhouette réelle à la silhouette perçue et test évaluant la personnalité), plus de la moitié ont été validés par au moins une étude et sont reconnus dans la recherche. Pour l'autre moitié, aucune information sur les qualités psychométriques n'est disponible.

\section{Tableau clinique}

Les informations recueillies dans cette catégorie concernent le poids, l'attitude face au poids et à l'image corporelle, la perception du corps et les distorsions cognitives, l'âge d'apparition du trouble et les comportements visant la perte de poids.

Poids. Le poids est abordé pour $65,5 \%$ des participants (15 articles, $\mathrm{N}=182$ ). Pour la majorité d'entre eux, on trouve des données indiquant soit l'indice de masse corporelle (IMC) (calculé à partir du poids et de la taille de l'individu selon la formule suivante : poids/ taille, un IMC moyen se situe entre 18.5 et 24.9), soit le poids pesé exprimé en pourcentage selon les normes attendues pour l'âge et la taille ou des données permettant d'en faire le calcul. Pour l'ensemble des participants pour lesquels l'IMC est disponible, la moyenne est de 16.1, ce qui, selon les normes d'interprétation de cet indice, les situent dans un état de dénutrition. Il est cependant possible que l'IMC moyen soit surestimé puisque l'un des articles n'indique que l'IMC maximal de son groupe d'adolescents. Concernant les articles qui présentent le poids en pourcentage, les participants sont en 
moyenne à 78,25 \% du poids attendu pour la taille et l'âge; ils se situent donc en deçà du poids attendu pour leur âge et leur taille (critère A du DSM-IV-TR).

Attitude quant au poids. L'attitude quant au poids est abordée pour $26,6 \%$ des participants (13 articles, $\mathrm{N}=74$ ). Les informations disponibles permettent d'estimer que $48,6 \%$ de ceuxci ont peur de prendre du poids et de devenir gros (critère B du DSM-IV-TR). Une comparaison avec un groupe contrôle indique que 40,5\% des participants ont obtenu un score supérieur à une échelle de l'Eating Disorders Inventory (EDI) mesurant le désir de minceur; la différence n'est cependant pas significative. Dans le même ordre d'idée, 46 \% des participants sont insatisfaits de leur poids et souhaitent en perdre. Une proportion de $6,8 \%$ des participants ciblés rapporte des attitudes envers leur poids différentes de celles mentionnées dans le DSM. Certains nient essayer de perdre du poids et d'autres adoptent des comportements de restrictions alimentaires pour atteindre un objectif autre que la perte de poids, par exemple pour développer leur masse musculaire, être en santé ou mourir.

Attitude quant à l'image corporelle. On dispose d'information concernant cette variable pour 33,1 \% des participants (9 articles, N=92). Dans l'ensemble, les participants se disent insatisfaits de leur corps ou éprouvent des inquiétudes liées à celui-ci. Une majorité, soit $61,9 \%$, mentionne que l'insatisfaction de leur image corporelle est liée à un désir d'avoir une masse musculaire plus importante tout en diminuant le gras corporel alors qu'une insatisfaction générale par rapport à leur corps est rapportée par 4,3\% d'entre eux. Lorsque comparés à un groupe contrôle, $32,6 \%$ des participants obtiennent un score significativement plus élevé à une échelle de l'EDI mesurant l'insatisfaction corporelle. 
Perception du corps et distorsions cognitives. Des données sont disponibles pour 21,2 \% (8 articles, $\mathrm{N}=59$ ) des participants et permettent de constater que $86,4 \%$ d'entre eux présentent une perception altérée de leur corps. Une comparaison entre les participants et un groupe contrôle indique que les adolescents qui souffrent d'anorexie surestiment significativement la taille de leurs épaules, de leurs hanches et de leurs cuisses. Un faible pourcentage des participants $(13,6 \%)$ a une perception adéquate de leur corps.

Âge d'apparition du trouble. L'âge d'apparition du trouble est abordé pour 50,7\% des participants (13 articles, $\mathrm{N}=141$ ). L'âge moyen d'apparition est estimé à 18,8 ans et varie entre 11 ans et 21 ans. Trois études ont fait une comparaison entre l'âge d'apparition du trouble chez leurs participants et celui de filles anorexiques et elles arrivent toutes à des conclusions différentes. La première conclut à un âge d'apparition plus tardif chez les garçons (20,1 ans comparativement à 18,5 ans), la seconde obtient des résultats indiquant que le trouble se développe plus tôt (16,5 ans comparativement à 18,7 ans) et la dernière n'obtient pas de différence significative entre les sexes (18,6 ans).

Comportements visant la perte de poids. Le type d'anorexie est spécifié pour 7,2\% des participants ( 4 articles, $\mathrm{N}=20$ ). De ceux-ci, $35 \%$ présentait une anorexie de type restrictif, $65 \%$ de type boulimique. Les stratégies mises en place pour perdre du poids et les différents comportements associés à l'AN sont quant à eux précisés pour 47,8\% des participants (14 articles, $\mathrm{N}=133$ ). Les comportements caractéristiques de l'AN-R (diètes, jeûnes et consommation d'aliments faibles en calories) sont rapportés par 20,3\% des participants. La pratique excessive d'exercice physique est quant à elle notée chez $60,2 \%$ 
des participants. Une importante consommation de liquide dans le but de masquer la sensation de faim est une pratique présente chez 14,3\% de l'échantillon. Les vomissements sont utilisés par 34,6\% des participants et les laxatifs par 27,8\% d'entre eux. Certaines autres caractéristiques de l'alimentation sont aussi mentionnées dans les articles. Parmi celles-ci on retrouve: des rituels précis autour de l'alimentation chez $37,6 \%$, le végétarisme / végétalisme chez $24 \%$ des participants, de l'anxiété liée au fait de manger en public ou un intérêt accru pour la nourriture et la confection des repas chez 9,8\%.

Parmi les études qui ont comparé les sujets garçons à des filles anorexiques sur la présence des comportements rapportés, certaines concluent à un tableau clinique global similaire entre les deux sexes alors que d'autres relèvent des différences liées à des comportements spécifiques. La divergence des résultats ainsi que les résultats contradictoires d'un article à l'autre complexifient les conclusions à tirer.

En résumé, ces résultats suggèrent chez les participants une plus grande prévalence de l'AN-B que de l'AN-R, une perception altérée de l'image corporelle et la présence d'insatisfaction quant au poids et au corps, particulièrement en ce qui concerne la musculature. Ces résultats doivent cependant être modérés par les faibles pourcentages de l'échantillon desquels ils sont issus. Les résultats sur lesquels il est possible de se prononcer avec plus de certitude en raison des proportions plus importantes de l'échantillon desquels ils sont tirés sont les suivants : les participants présentent un poids en deçà du poids santé, leur âge d'apparition moyen se situe aux environs de 18,8 ans et leurs comportements visant la perte de poids sont l'exercice physique excessif suivi par les vomissements, l'usage de laxatifs et les restrictions alimentaires. 


\section{Caractéristiques préalables au trouble}

Des données sur les caractéristiques présentes avant ou au moment de l'apparition du trouble sont fournies pour 32,4\% de l'échantillon (18 articles, $\mathrm{N}=90)$.

Médicales. Des difficultés d'ordre physique sont rapportées chez $10 \%$ d'entre eux. Pour $3,3 \%$, celles-ci se sont manifestées à peu près en même temps que le début des pertes de poids (problèmes de santé et gastroplasties en raison d'une obésité, tous avant l'âge de 30 ans). Des retards développementaux ont quant à eux été relevés chez 5,6\% des participants.

Poids. De l'obésité prémorbide est rapportée pour $14,4 \%$ des participants et un poids supérieur au poids attendu chez 4,4\%. Elle serait plus caractéristique de ceux atteints de boulimie.

Sociales. On note que $15,6 \%$ des participants ont été victimes de moqueries ou d'intimidation par leurs pairs (en raison de leur apparence physique, de leur homosexualité, d'une identité de genre confuse ou de difficultés scolaires) et que 3,3\% ont souffert ou souffrent d'isolement social. Pour certains, les commentaires des pairs sur leur poids sont décrits comme des facteurs ayant précipité le début des diètes.

Antécédents familiaux. Des antécédents familiaux d'obésité ou de surpoids sont présents chez $25 \%$ des participants. La présence d'antécédents psychologiques familiaux est rapportée chez 28,9\% des participants (trouble obsessionnel compulsif, AN, alcoolisme). En ce qui concerne le fonctionnement familial, des conflits sont rapportés entre le sujet et les autres membres de la famille dans $12,2 \%$ des cas. Certains auteurs, qui n'appuient pas 
leur conclusion par des chiffres, notent chez leurs participants une propension à l'enchevêtrement des liens familiaux ainsi qu'à l'évitement des conflits. Dans $11,1 \%$ des cas, des séparations, des pertes ou l'absence d'un membre de la famille sont rapportés. On note enfin chez $3,3 \%$ des participants des familles contrôlantes, compétitives ou avec de grandes attentes pour leur fils.

Psychopathologie. Les troubles les plus fréquemment rapportés chez les participants avant l'épisode d'anorexie sont ceux évoquant des processus psychotiques. En effet, ceux-ci sont évoqués pour $14,4 \%$ des participants. Une proportion de $4,4 \%$ a présenté des antécédents de difficultés alimentaires durant l'enfance. Chez 6,7 \%, des difficultés soit au niveau de la discipline, soit des comportements agressifs, soit des symptômes du TDA/H ou dépressifs ont été notés.

La synthèse des résultats concernant les caractéristiques préalables au trouble souligne chez les participants la présence d'un surplus de poids, d'intimidation par les pairs, des troubles de nature psychotiques avant le début de l'épisode d'AN et la présence d'antécédents familiaux de psychopathologies et d'obésité. Les résultats ne sont cependant, issus que d'un tiers de l'échantillon.

\section{Troubles comorbides}

La question de la comorbidité est abordée pour 28,8 \% de l'échantillon (15 articles, $\mathrm{N}=80$ ). Le trouble le plus fréquemment mentionné comme concomitant à l'anorexie est la dépression. Les auteurs rapportent un diagnostic de dépression ou des caractéristiques permettant d'inférer celui-ci pour $27,5 \%$ des participants. Le second trouble le plus 
fréquent est l'abus de substance identifié chez 11,25\% des participants. Les autres troubles comorbides mentionnés sont des caractéristiques obsessionnelles $(17,7 \%)$ et les troubles de la personnalité répartie dans les trois Clusters du DSM (7,7\%).

Ces résultats suggèrent donc une association de l'AN à la dépression et à l'abus de substance, ainsi qu'à la présence de caractéristiques obsessionnelles.

\section{Orientation sexuelle}

L'orientation sexuelle est considérée pour 19,4\% des participants (11 articles, 54 sujets). De ceux-ci, 25,9\% sont décrits comme étant hétérosexuels et $13 \%$ homosexuels. La présence d'asexualité, définie par les auteurs comme «l'absence d'intérêt sexuel pour l'un ou l'autre des deux sexes depuis au moins un an », est aussi relevée pour 35,2\% des participants. Toutefois, ces études ne mentionnent pas s'ils avaient déjà eu de l'intérêt sexuel pour l'un ou l'autre des deux sexes. Cela aurait pu être pertinent puisque le désintérêt pour la sexualité a déjà été attribué par des chercheurs aux conséquences hormonales des restrictions alimentaires. Pour 25,9\% des participants, l'information fournie sur l'orientation sexuelle ne permet pas d'identifier celle-ci.

Les résultats suggèrent donc une proportion importante d'homosexualité et d'asexualité chez les participants. Il faut cependant considérer qu'ils ne sont issus que de 19,4\% de 1'échantillon.

\section{Activité sexuelle}

De l'information sur l'activité sexuelle est présentée chez $11,2 \%$ des participants (5 articles, $\mathrm{N}=31$ ). De ceux-ci, les articles mentionnent que $9,7 \%$ sont ou ont déjà été 
sexuellement actifs et que $12,9 \%$ n'ont jamais eu de relation sexuelle. Selon les auteurs, $22,6 \%$ des participants auraient entretenu des attitudes sexuelles prémorbides anormales (manque d'intérêt sexuel, peur ou aversion des contacts physiques avec l'autre sexe ou promiscuité sexuelle). Pour 45,2\% des participants, l'information disponible est insuffisante pour déterminer si les participants étaient actifs sexuellement.

En raison du faible pourcentage de l'échantillon duquel les données sont issues, du nombre important de données manquantes, de l'absence de définition claire et précise des concepts ainsi que du manque d'information nécessaire pour modérer les résultats en fonction de l'âge des participants, il est difficile de tirer des conclusions pour cette variable.

\section{Identité de genre}

Des données sur l'identité de genre sont disponibles pour $5 \%$ des participants (5 articles, $\mathrm{N}=14$ ). Pour 21,4\% d'entre eux, les auteurs concluent à la présence d'un trouble de l'identité de genre. Des particularités sont présentes à ce niveau chez $71,4 \%$ des participants, mais elles sont insuffisantes pour permettre de conclure à un trouble de ce type. Parmi celles-ci, on note la présence de masculinité conflictuelle (discordance entre l'identité de genre consciente et inconsciente), insatisfaction quant au genre et confusion quant à l'identité de genre. L'identité de genre ne semble pas poser problème $7,1 \%$ des participants.

Les résultats suggèrent donc des troubles ou des particularités au niveau de l'identité de genre pour la majorité des participants, cependant le petit nombre de participants sur lesquels porte cette conclusion la rend fragile. 


\section{Personnalité}

Les caractéristiques de la personnalité sont traitées pour $28,4 \%$ des participants (10 articles, 79 sujets). Les résultats sont issus de recherches adoptant des approches théoriques ainsi que des méthodologies et instruments variés. Dans le but de favoriser une mise en commun des résultats, et ce malgré les limites que cela peut représenter, ils ont été regroupés en trois catégories: structure de personnalité et mécanismes de défense, représentation d'objet et trait de personnalité. Une bonne partie des articles qui traitent des aspects portant sur la personnalité n'aborde qu'une de ces caractéristiques. Ainsi, les données sur ces aspects sont partielles et ne permettent pas une répartition complète des participants pour chacune des caractéristiques.

Structure de personnalité et mécanisme de défense. La structure de personnalité et les mécanismes de défense sont abordés pour $38 \%$ des participants concernés par cette variable. La structure de personnalité psychotique est celle qui semble présente chez le plus grand nombre de participants avec un taux de $15,1 \%$ pour lesquels de l'information est disponible. Une structure névrotique est suggérée chez 3,8\% des participants tandis que la personnalité limite est présente chez 1,2\%. La structure de personnalité a été évaluée cliniquement par entrevue; aucune comparaison à des normes n'est disponible dans les études. La structure de personnalité n'ayant pas été étudiée systématiquement, aucune information n'est disponible pour les 79,9\% restant. Pour ce qui est des mécanismes de défense, un faible niveau d'expression de l'anxiété et de l'angoisse et une faible utilisation de la rationalisation sont rapportés chez 13,9\% des participants. D'autres notent chez leurs 
participants $(2,5 \%)$ des défenses variées ainsi que des défenses d'intellectualisation en réponse aux émotions de colère. Les mécanismes de défense ont été évalués à l'aide d'observations cliniques et de mesures objectives et projectives (Defensive Style Questionnaire 40 et Rorschach).

Représentation d'objet. La représentation d'objet est abordée pour 10,1\% des participants. Elle a été évaluée à l'aide du Assessment of qualitative and structural dimension of object representation, un outil permettant de situer la représentation d'objet sur un continuum allant d'une représentation précoce à mature. Contrairement aux attentes des auteurs, il semble que les participants se situent dans la norme en ce qui a trait au niveau de relation d'objet. Les résultats sont cependant hétérogènes à l'intérieur de cet échantillon restreint.

Traits de personnalité. Les traits de personnalités sont abordés pour $53,2 \%$ des participants concernés par cette variable et ont été évalués à partir d'observations cliniques ainsi que de résultats à des outils d'évaluation (Temperament and character inventory et Bender motor gestalt). Chez 12,7\% d'entre eux, on note la présence de traits obsessionnels, compulsifs ou rigides. L'évaluation de $35,7 \%$ des participants révèle, quant à elle, une tendance à maintenir un comportement engendrant des frustrations, une faiblesse au niveau l'acceptation de soi et de la capacité à diriger ses comportements vers des objectifs. La présence de traits antisociaux et explosifs a aussi été notée.

De ces résultats, la présence de structure de personnalité psychotique ressort comme la caractéristique importante chez les participants. Certains traits de personnalités sont aussi relevés pour une proportion considérable des participants. Cependant, le fait qu'il s'agisse 
de tendances pour lesquelles aucun chiffre n'est fourni empêche d'en apprécier l'importance.

\section{Pronostic}

La question du pronostic de l'anorexie est abordée pour $12.9 \%$ des participants de l'échantillon (14 articles, $\mathrm{N}=36$ ). Un pronostic favorable est rapporté pour environ $88 \%$ des participants. Cependant, des données de suivi post-traitement (un mois à deux ans) ne sont disponibles que pour 30,5\% d'entre eux. Elles indiquent qu'il y aurait un maintien des améliorations quant au poids et aux comportements alimentaires après le traitement et, pour certains, on mentionne aussi des progrès dans d'autres sphères de leur vie. Pour les 58,3 \% restant, une évolution favorable des comportements et des symptômes anorexiques est rapportée. Par contre, les conclusions doivent être modérées par l'absence de suivis et par le fait que pour une majorité $(44,4 \%)$, les auteurs soulignent que le contexte de vie demeure problématique, ce qui augmente les risques que le trouble revienne. Pour $11,1 \%$ des participants, les auteurs rapportent des informations permettant de conclure que le trouble persiste. Parmi celles-ci, on retrouve la persistance de préoccupations envahissantes pour le poids, des restrictions alimentaires, l'arrêt précoce des traitements ou la perte de poids avant la fin du traitement.

Ces résultats, malgré leurs limites et le nombre restreint de participants sur lesquels ils portent, suggèreraient donc un pronostic favorable pour les garçons atteints d'AN. 


\section{Discussion}

Dans cette section, nous avons choisi de regrouper les résultats de certaines variables à l'étude dans la recension en catégories afin d'en faciliter la discussion. Elles ont été groupées sous les rubriques suivantes: portrait clinique, caractéristiques psychologiques, orientation sexuelle et identité de genre.

Concordance avec la recherche passée. La section précédente permet de constater qu'actuellement les écrits scientifiques portant sur l'AN chez les adolescents et jeunes adultes de sexe masculin permettent de relever certaines tendances, mais qu'il est encore difficile de se prononcer avec certitude sur les différentes caractéristiques associées à ce trouble chez ce groupe. Les résultats de la recension, malgré la limite de leur portée, vont tout de même dans le sens de plusieurs constats émis par les recherches passées (Andersen, 1999; Chambry, 2002; Fichter, 1995; Herzog, 1984; Romeo, 1994). Parmi ceux-ci on retrouve une proportion plus importante d'AN-B que d'AN-R, un âge d'apparition similaire à celui des filles, l'importance de la présence de l'obésité prémorbide et de la confusion au niveau de l'identité de genre, des taux élevés d'homosexualité et d'asexualité ainsi que des aspects de la personnalité caractéristiques d'une structure psychotique.

\section{Portrait clinique}

L'analyse des résultats disponibles suggère que les garçons atteints d'anorexie présentent des pertes de poids correspondant au critère A du DSM-IV-TR, c'est-à-dire qui sont assez importantes pour être qualifiées de cliniquement significatives. En effet, les poids moyens des garçons adolescents et adultes dans les études sont inférieurs à l'IMC minimal de 18.5 
ou à $85 \%$ du poids minimal attendu pour la taille et l'âge. Ces résultats ne surprennent guère puisque la majorité des participants a été recrutée dans des milieux hospitaliers suite à un traitement en clinique interne ou externe pour AN. Ce constat assure une certaine validité quant au diagnostic posé chez les participants des études, mais impose de limiter nos conclusions aux garçons souffrant d'une anorexie ayant atteint un degré considérable de sévérité (c.-à-d. nécessitant un suivi en milieu hospitalier). On ne peut affirmer donc que les garçons qui n'ont pas été pris en charge dans ce type de services (possiblement spécialisés) présentent les mêmes caractéristiques au niveau du poids. Cette remarque est aussi valable pour les garçons qui n'ont pas été dépistés ou encore qui ne satisfont pas tous les critères diagnostiques de l'AN (donc qui se situent à un seuil sous-clinique). S'il y a en effet une différence dans l'intensité des pertes de poids entre le groupe représenté par l'échantillon de la recension et les garçons atteints d'une AN d'une sévérité moindre, cela pourrait expliquer pourquoi le diagnostic d'AN ne serait posé que lorsqu'un degré de sévérité important a été atteint, comme l'ont suggéré plusieurs auteurs (Andersen, 1999; Bramon-Bosch, 2000; Crosscope-Happel, 2000; Lock, 2009; Muise, 2003; Peebles, 2007). Puisque les données disponibles pour la variable poids touchent un pourcentage élevé de l'échantillon, soit $65,5 \%$, cette conclusion peut être émise avec plus de certitude que certaines autres. Les résultats analysés ne fournissent cependant pas ou peu d'information quant au rythme et aux pourcentages de la perte de poids. Dans le passé, certaines études ont affirmé que les garçons perdaient un pourcentage important de leur poids (Herzog, 1984), alors que d'autres ont affirmé l'inverse (Fichter, 1985). Poursuivre les investigations sur cet aspect du portrait clinique pourrait être pertinent à l'avenir, entre autres pour 
favoriser une meilleure reconnaissance du trouble chez cette population. En effet, si le constat que les garçons perdent un pourcentage moins important de leur poids ou le perde moins rapidement était fait, le dépistage du trouble devrait prendre en compte d'autres critères que le poids. Par ailleurs la confirmation d'une perte de poids importante inciterait à favoriser un dépistage précoce.

Les conclusions sur les autres critères diagnostiques sont tirées de proportions moins importantes d'informations sur les participants, mais suggèrent tout de même une correspondance. En effet, comme il a déjà été suggéré par certains auteurs, la recension soulève que les participants garçons atteints d'AN sont insatisfaits de leur poids et souhaitent en perdre, ils ont peur de devenir gros et présentent une perception altérée de leur corps. Les résultats de quelques études notent aussi des similarités entre les garçons et les filles quant à la fréquence et à l'intensité des restrictions alimentaires, à l'utilisation des vomissements, des crises de boulimies et de l'exercice excessif. Cependant, d'autres auteurs observent des différences sur ces mêmes comportements, telles un recours plus fréquent à l'exercice physique comme moyen de contrôle du poids et une moindre utilisation des laxatifs chez les garçons. Les constats des études comparant les garçons et les filles ne sont donc pas consensuels. Les différences dans les résultats pourraient être attribuables aux caractéristiques des études incluant la constitution des échantillons en ce qui a trait à l'âge des participants et au type d'AN dont ils sont atteints. Le manque de consensus pourrait aussi être lié à l'évaluation des participants à différents moments dans l'évolution de leur trouble. Dans ce cas, dans des recherches futures il serait pertinent de recueillir de l'information sur le type d'AN ainsi que sur la durée du trouble présenté par 
les participants pour permettre l'obtention de données plus comparables et ainsi favoriser la possibilité d'obtenir un meilleur consensus. Concernant la grande utilisation de l'exercice physique, certains auteurs ont émis l'hypothèse que l'hyperactivité physique serait une stratégie socialement acceptable pour perdre du poids chez les garçons, ce qui pourrait favoriser son utilisation et faire en sorte qu'une grande implication dans des activités sportives pourrait passer plus inaperçue.

Outre le critère concernant l'aménorrhée, il semble donc qu'il y ait des similarités entre les garçons et les filles quant aux symptômes décrits par le DSM. Les participants sont cependant atteints d'une AN pouvant être qualifiée de sévère. Comme l'ont mentionné certains auteurs dans le passé, cela pourrait avoir pour conséquence de minimiser les différences possibles entre les sexes dans des présentations cliniques plus légères (Woodside, et al., 2001). Il est aussi important de considérer que le DSM ne fournit qu'une liste de symptômes observables et qu'ainsi, une similarité avec les filles sur les aspects comportementaux du trouble ne permet pas de conclure à une telle similarité au plan intrapsychique ou sur d'autres aspects du trouble inexplorés jusqu'à présent chez les garçons.

Comme il a déjà été constaté dans des études antérieures, la recension obtient des résultats indiquant que les garçons sont insatisfaits de leur image corporelle. Il semble cependant qu'ils le sont pour des raisons différentes de celles exprimées par les filles. L'insatisfaction de l'image corporelle chez les garçons est plus particulièrement liée à la masse musculaire qu'au poids. Les garçons souhaitent augmenter leur masse musculaire et leur perte de poids est motivée par cet objectif. Cette motivation peut être associée aux 
standards de beauté masculine véhiculés par la société (Harvey \& Robinson, 2003). Les résultats de la recension soulèvent la présence importante d'obésité prémorbide et de surplus de poids chez les garçons anorexiques ainsi que dans leur famille. On peut poser l'hypothèse selon laquelle pour certains garçons, l'insatisfaction de l'image corporelle proviendrait d'une réelle discordance entre l'image corporelle et l'idéal social, ce qui les mettrait ainsi plus à risque de développer un TCA. La présence d'une insatisfaction de l'image corporelle étant considérée par certains auteurs comme un facteur prédisposant au développement du trouble (Stice \& Shaw, 2002). De plus, les résultats de la recension suggèrent qu'une proportion considérable des garçons atteints d'AN ont déjà été victimes d'intimidation par leurs pairs, entre autres en lien avec leur apparence physique. Ces moqueries ont donc possiblement contribué au développement de l'insatisfaction par rapport au corps. Toutefois, le fait que l'AN transforme le corps des garçons d'une manière qui finit par les éloigner de leur idéal musculaire met en lumière la nécessité de se pencher plus longuement sur l'étiologie du trouble puisque celle-ci ne peut, à notre avis, être uniquement restreinte au désir d'atteindre un idéal social.

La recension fournit aussi certaines pistes de réflexion sur des caractéristiques du portrait clinique autres que celles évoquées dans le DSM. Les résultats rapportent un nombre plus important de garçons atteints d'AN-B que d'AN-R, ce qui nous impose d'associer l'ensemble des résultats plus spécifiquement à cette population. Il ressort aussi que la comorbidité la plus fréquente est la dépression suivie de l'abus de substance. La présence de traits de personnalité antisociaux et explosifs chez plusieurs garçons est aussi mise en évidence (Fassino, 2001). Cela pourrait ouvrir un questionnement à savoir si les 
garçons auraient un profil similaire aux filles boulimiques chez qui la présence d'impulsivité serait plus caractéristique et dont le trouble serait plus fréquemment associé à l'abus de substance et à d'autres troubles du contrôle des impulsions (Bryant-Waugh, 2007; Reijonen 2003). Nous pourrions aussi nous demander si, comme l'ont suggéré certains, la problématique des garçons anorexiques ne s'inscrirait pas dans un ensemble plus large de troubles des conduites (Chambry \& Agman, 2006). Cependant, considérant les difficultés diagnostiques caractéristiques des garçons, il est possible que l'anorexie soit plus fréquemment dépistée chez des garçons présentant aussi des troubles externalisés. Les caractéristiques plus visibles de ces derniers ayant attiré l'attention de l'entourage et des intervenants sur la nécessité de consulter. À ce stade, nous ne pouvons cependant parler que d'hypothèses puisque le support empirique à de telles affirmations est largement insuffisant. Pour tenter d'aller plus loin dans ce type d'hypothèses concernant une plus grande association à des troubles des conduites ainsi qu'une similarité avec les filles boulimiques, il serait nécessaire d'effectuer des études auprès d'échantillons cliniques des deux sexes, contrôlant le type d'anorexie et dans lesquelles des mesures comportementales et de personnalité seraient incluses. Ce type d'études permettrait de déceler de possibles différences ou similarités entre les différents sous-groupes (garçons atteints d'AN-R et d'AN-B et filles anorexiques des mêmes types).

Deux autres pistes de réflexion sont aussi suggérées, mais doivent être nuancées en raison de certaines failles méthodologiques notées dans les études de la recension. La première est qu'il semble que les garçons aient un âge d'apparition du trouble qui se situe à 18,8 ans. Il s'agit ici d'un âge moyen issu d'un échantillon d'adolescents et de jeunes 
adultes, ce qui a pu faire en sorte d'introduire une plus grande variabilité dans les résultats et de créer un biais au niveau du rappel exact de l'âge d'apparition, particulièrement pour les participants plus âgés dont le début du trouble peut remonter à plusieurs années. Les résultats de recherches pourraient donc être biaisés dans une certaine mesure. Ils sont d'ailleurs variables d'un article à l'autre. Il faut aussi mentionner que dans plusieurs études, l'âge d'apparition ne semble pas avoir été contrôlé avec rigueur. En effet, il est parfois difficile de savoir si les auteurs rapportent l'âge d'apparition du trouble ou s'ils font plutôt référence à l'âge auquel le sujet est arrivé dans les services. Dans les recherches futures, il serait souhaitable d'étudier de manière plus précise l'âge auquel les premiers symptômes sont apparus. Une meilleure connaissance de ce dernier pourrait entre autres être utile pour mieux déterminer la clientèle à cibler par les programmes de prévention. L'âge d'apparition souligné dans la recension est comparable au deuxième pic d'âge d'apparition suggéré pour les filles dans les écrits qui est de 18 ans (le premier étant de 14 ans).

La seconde piste de réflexion est qu'il semble que les garçons ont un pronostic très favorable, ce qui va à l'encontre de ce qui avait été suggéré dans les recherches antérieures. Ce résultat doit cependant être modéré par le fait qu'il est issu d'un très faible pourcentage de participants de l'échantillon total, pour lesquels on ne dispose que de peu de données de suivi post-traitement. Il est donc difficile de considérer ces résultats comme valides. Une étude suggère cependant une piste de recherche intéressante selon laquelle chez les garçons anorexiques, l'impulsivité et le fait d'avoir été sexuellement actif avant le début du trouble seraient des facteurs qui semblent influencer favorablement le pronostic. 
Caractéristiques psychologiques de l'anorexie chez les garçons

Les résultats de la recension suggèrent qu'un taux considérable des participants a déjà vécu différents troubles évoquant des processus psychotiques avant l'épisode d'AN ou présente une structure de personnalité qualifiée de psychotique. Les auteurs de l'un des articles évoquent même la possibilité que les conduites restrictives permettent de maintenir un contrôle sur les tendances à la défragmentation psychique (Daini, 2008). Ces constats vont dans le sens de ce qui est parfois énoncé dans les écrits scientifiques, soit que les garçons anorexiques seraient atteints d'une pathologie générale plus lourde que les filles (Bercé, 2009). Cela ouvre la porte à des hypothèses sur la possibilité que l'anorexie soit, pour certains garçons, un prodrome d'un trouble de nature psychotique ou schizophrénique. Cela pourrait en partie expliquer la nature complexe de l'anorexie chez les garçons et pourrait être appuyé par le fait qu'il semble que les garçons ont des antécédents psychologiques familiaux plus sévères que les filles. Les résultats concernant les antécédents de nature psychotique doivent cependant être modérés puisqu'ils ne sont issus que d'un peu plus du quart de l'échantillon et d'études ayant recours à des méthodologies très variables. Tout ceci suggère tout de même que le tableau clinique d'AN reste une manifestation de symptômes observables, n'impliquant pas nécessairement un fonctionnement psychique identique chez tous.

Orientation sexuelle et identité de genre

Les résultats de la recension indiquent un taux d'homosexualité de $13 \%$. Bien qu'il soit inférieur à ce qui a déjà été rapporté dans le passé (entre $20 \%$ et $58 \%$ ), il s'agit d'un taux largement supérieur à celui rapporté pour la population générale et pour les filles 
anorexiques (4 à $5 \%$ ). Malgré que certains estiment les connaissances actuelles insuffisantes pour établir l'homosexualité comme un facteur de risque pour l'anorexie (Striegel-Moore \& Bulik, 2007), cette particularité a engendré la formulation de plusieurs hypothèses quant au lien entre l'homosexualité et l'anorexie. Parmi celles-ci, on retrouve la possibilité que la valorisation de l'apparence physique caractéristique des communautés homosexuelles puisse aggraver le trouble une fois que celui-ci est présent et engendrer une surreprésentation des patients homosexuels dans les unités de traitement. D'autres hypothèses portent quant à elles sur des enjeux plus profonds. Plusieurs ont suggéré que l'anorexie aurait une valeur défensive contre des mouvements pulsionnels inconfortables. Ainsi, il pourrait s'agir d'un moyen de retarder les enjeux sexuels pour des individus à l'orientation homosexuelle conflictuelle. Bien que les résultats de la recension ne nous permettent pas de l'appuyer, il est intéressant de constater que certains auteurs ont déjà relevé que le pronostic de leur sujet était plus favorable si ces derniers avaient déjà eu des relations sexuelles avant le début de leur trouble. Ce constat est cohérent avec l'hypothèse émise plus tôt voulant que l'anorexie puisse inconsciemment retarder des pulsions sexuelles conflictuelles. On peut en effet inférer que le fait d'avoir été actif sexuellement serait un signe d'une orientation sexuelle moins conflictuelle. Compte tenu de la présence de telles hypothèses dans les écrits sur le sujet, il serait pertinent de poursuivre cette piste de recherche et d'examiner plus systématiquement l'activité sexuelle préalable au trouble chez les garçons atteints d'anorexie.

Il est toutefois à noter que toutes les recherches ne comptent pas des proportions aussi substantielles d'homosexualité chez leurs participants et que, comme le soulignent 
certains, il est possible que des stéréotypes entretenus sur le lien entre l'anorexie et l'homosexualité puissent influencer l'interprétation de résultats plus ambigus (Bercé, 2009). Il est toutefois nécessaire de poursuivre l'exploration de l'orientation sexuelle et des liens possibles avec l'AN. Les résultats de la recension indiquent aussi qu'une proportion considérable de participants pouvait être qualifiée d' "asexuels » (absence d'intérêt sexuelle pour l'un ou l'autre des deux sexes depuis au moins un an).

Dans le cadre de cette recension, nous avons constaté que dans certaines études la manière dont l'information a été récoltée n'est pas explicite, que l'orientation sexuelle ne semble pas avoir été rigoureusement contrôlée et que dans certains cas, elle n'est rapportée que pour une partie des participants à l'étude. Il semble aussi que dans certains cas, des préjugés aient pu interférer avec l'obtention de résultats objectifs quant à l'orientation sexuelle (ex : inclure le fait de ne pas avoir d'intérêt sexuel pour l'autre sexe dans une catégorie nommée «attitudes sexuelles anormales »). Le constat selon lequel des failles dans les méthodologies des études complexifient l'obtention d'un portrait fidèle de l'orientation sexuelle chez les garçons anorexiques a d'ailleurs déjà été fait par d'autres dans le passé (Andersen, 1999). À l'avenir, il serait donc souhaitable de formuler les questions sur l'orientation sexuelle de manière non équivoque, neutre et d'étudier cette variable de manière plus systématique. Il serait aussi souhaitable que les auteurs prennent en compte la possibilité de l'absence d'intérêt sexuel momentanée. Il serait ainsi utile de trouver une manière de situer l'orientation sexuelle sur un continuum plutôt que de la classifier de manière dichotomique. Cela permettrait entre autres de garder en tête que l'orientation sexuelle peut être un concept plus dynamique que statique, particulièrement 
durant l'adolescence. Pour ce faire, il serait cependant nécessaire de mieux opérationnaliser le concept «d'asexualité » émis par certains auteurs pour permettre une réplique des recherches (ex. : en fonctions de l'intérêt sexuel, de l'activité sexuelle et de la durée). Cette poursuite de la recherche sur l'orientation sexuelle est particulièrement pertinente en raison des différentes hypothèses quant aux liens possibles entre l'homosexualité et l'anorexie proposées dans les écrits ainsi qu'en raison de la possibilité soulevée par certains que les restrictions alimentaires pourraient avoir comme conséquence une diminution de la testostérone et donc influencer l'intérêt pour la sexualité. Il faut cependant garder en tête qu'il peut être délicat d'évaluer l'orientation sexuelle chez des adolescents, puisqu'il est possible qu'elle soit incertaine pour ces derniers ou changeante au fil des expériences de vie. Cela pourrait entre autres contribuer à la variabilité des résultats pour cette population.

Ces hypothèses concernant l'orientation sexuelle sont souvent accompagnées d'autres questionnements liés à l'identité de genre. Les résultats soulèvent en effet que $92,8 \%$ des participants pour lesquels cette variable a été examinée ( $5 \%$ du $\mathrm{N}$ total) présente un trouble de l'identité de genre ou du moins, certaines caractéristiques associées à ce dernier. L'un des auteurs note que ce sont les participants qui présentent le plus de discordance entre l'identité consciente et inconsciente qui sont les plus sévèrement atteints par l'AN et suggère que cette incongruité pourrait jouer un rôle dans l'AN (Bassett, 2002). Dans ces cas, certains croient que les conduites restrictives pourraient servir à supprimer les caractéristiques sexuelles masculines dans le but de s'approcher des modèles de beauté féminins ou à fuir les enjeux liés à la maturité sexuelle qui sont une source d'anxiété. Des hypothèses cliniques ont d'ailleurs été formulées à l'effet que les difficultés d'identité de 
genre seraient issues d'une identification importante à la mère, engendrée par un père absent ou en raison d'une relation distante avec ce dernier et que l'identification au sexe féminin pourrait avoir pour fonction de préserver un lien d'attachement fondé sur l'identique avec la mère. Il ne semble cependant pas y avoir d'appui empirique à ces dernières. La question d'une grande proximité avec la mère soulève aussi la possibilité d'un conflit œdipien non résolu contre lequel la modification des comportements alimentaires pourrait tenter d'avoir une influence. Cette modification pourrait viser la diminution des pulsions libidinales inacceptables pour le sujet dans le but de maintenir la relation parentale. Nous pourrions aussi poser l'hypothèse qu'un repli sur l'homosexualité ou encore l'asexualité pourrait être un moyen de mise à distance de ce conflit. Il faut cependant garder en tête que ces hypothèses ne sont pas supportées empiriquement et que l'adolescence est accompagnée d'enjeux psychiques que certains garçons pourraient tenter d'éviter par une restriction calorique soit parce qu'ils les perçoivent comme trop menaçants ou parce qu'ils ne se sentent pas suffisamment prêts psychiquement pour les affronter. Il serait souhaitable de poursuivre l'investigation de l'identité de genre chez les garçons anorexiques sur un nombre plus important de sujets que ce qui a été fait jusqu'à présent. Il est aussi primordial que les chercheurs fournissent des définitions claires des concepts qu'ils utilisent afin de mettre fin à la confusion qui semble actuellement présente dans les écrits scientifiques quant au concept de conflit au niveau de l'identité de genre.

\section{Limites des résultats}

La difficulté d'obtenir des consensus et de se prononcer avec certitudes sur les caractéristiques associées à l'anorexie masculine peut, en partie être attribuable aux 
méthodologies des études dont certaines caractéristiques limitent les résultats de la recension.

D'abord, il existe un grand écart d'âge entre les participants. Il s'agit d'une limite importante puisque comme l'a mentionné Muise (2003) pour la prévalence du trouble, il est possible que l'âge des participants ait un impact sur différentes caractéristiques associées à l'anorexie. Le fait que la presque totalité $(99,3 \%)$ des participants a été recrutée en milieu hospitalier limite aussi les résultats à cette population spécifique et empêche de les généraliser à des garçons atteints d'une forme moins sévère d'anorexie.

Ensuite, la grande variété d'instruments psychométriques utilisés ainsi que la pluralité des variables ciblées par les études rendent difficiles les comparaisons entre ces dernières pour un nombre significatif de participants. En effet, bien que certaines variables sont abordées pour un pourcentage important de l'échantillon, d'autres ne le sont que pour de faibles pourcentages. Ce qui fait que, somme toute, les conclusions de la recension sont pour la plupart appuyées sur un nombre restreint de participants. Une autre problématique liée aux instruments psychométriques est que certains ont originalement été conçus pour l'évaluation de filles et bien que les auteurs les aient adaptés pour les utiliser auprès des garçons, les modifications n’ont pas été validées empiriquement. Il est aussi à noter que plusieurs auteurs fournissent peu de précisions quant à la manière dont la collecte de certaines informations a été effectuée et aux critères utilisés pour l'évaluation des variables.

Par ailleurs, le fait que certaines variables n'ont pas été abordées de manière systématique (ex.: orientation sexuelle, identité de genre) ouvre la porte à plusieurs questionnements lorsque nous sommes confrontés à un manque d'informations pour un 
sujet donné. Par exemple l'absence d'information peut être interprétée de manières différentes : soit la variable n'a pas été contrôlée, soit aucune particularité n'a été relevée pour le sujet.

Enfin, le fait que les résultats des études incluses dans la recension n'aient pas été pondérés en fonction de leur qualité méthodologique représente une limite puisque le même poids est accordé aux résultats évalués subjectivement par les chercheurs qu'à ceux issus d'analyses plus rigoureuses ou soumis à une comparaison avec des normes établies. Cependant, dans le cadre de la présente recension, qui visait à regrouper des informations portant sur différentes caractéristiques du tableau clinique et psychologique, la variabilité des méthodologies et des variables à l'étude ont rendu une telle pondération impossible. Une dernière limite est que le choix des articles à inclure dans la recension a été limité par la langue ainsi que par la base de données utilisée. Il n'est donc pas à exclure que d'autres études traitant de l'anorexie chez les garçons adolescents et jeunes adultes existent, mais n’ont pas été incluses dans la recension.

\section{Piste et suggestion pour la recherche future}

Sur la base de la recension des écrits effectuée sur l'anorexie chez les garçons, certaines recommandations peuvent être émises pour les recherches futures. La première est qu'il semble maintenant assez bien établi que lorsqu'il est évalué avec les outils originalement conçus pour les filles, le portrait clinique comportemental de l'anorexie chez les garçons est similaire à celui des filles sauf pour quelques comportements. Pour faire avancer les connaissances propres aux garçons, il serait souhaitable que des efforts soient mis pour développer des outils de mesures spécifiques aux garçons. Même si les 
symptômes semblent très similaires, la création d'instruments de mesure plus adaptés aux populations masculines pourrait permettre d'explorer plus en profondeur les motivations sous-jacentes aux symptômes qui semblent différer entre les sexes. Dans le même ordre d'idée, dans le but de permettre l'identification des caractéristiques propres aux garçons, il serait intéressant que les recherches adoptent un modèle d'observations cliniques plutôt que de comparer d'emblée les garçons aux connaissances déjà établies sur les filles atteintes d'anorexie. Cela pourrait faciliter l'identification des caractéristiques qui auraient pu passer inaperçues en raison d'une attention particulièrement ciblée sur les caractéristiques habituellement retrouvées chez les filles.

Il est aussi important que les chercheurs dans ce domaine ciblent de manière plus précise certaines variables du fonctionnement psychologique qu'ils souhaitent approfondir (ex. : caractéristiques de la personnalité, enjeux liés à la sexualité, relation d'objet, identité de genre, etc.). Cela serait nécessaire dans la mesure où nous disposons de différentes pistes de réflexion et que plusieurs hypothèses sont formulées dans les écrits quant aux possibles liens entre le fonctionnement psychologique et l'anorexie. Celles-ci manquent toutefois encore de support empirique.

Sur le plan méthodologique, plusieurs éléments pourraient améliorer la qualité et la quantité d'informations dont nous disposons chez les garçons. D'abord, il serait primordial de systématiser la quête d'informations sur des variables qui, bien qu'encore mitigées dans les écrits scientifiques, ressortent comme importantes, par exemple l'homosexualité, l'identité de genre, les fragilités psychiques, les comorbidités et l'activité sexuelle. Il serait aussi nécessaire que les auteurs fournissent des définitions claires des concepts qu'ils 
évaluent pour permettre de bonnes reproductions des études ainsi que la comparaison de leurs résultats. Ensuite, il serait intéressant d'avoir accès à des études effectuées auprès d'échantillons d'âges moins variables (ex : groupes composés d'adolescents exclusivement) et d'échantillons composés de garçons provenant de services similaires (uniquement externe ou uniquement interne). L'utilisation de groupes contrôles composés de garçons de la population générale ou atteints d'autres troubles psychologiques pourrait être pertinente pour permettre de mettre en relief ce qui distingue les garçons anorexiques des autres garçons. En effet, il semble que la fréquente utilisation de groupes de comparaisons composés de filles anorexiques peut biaiser la quête d'informations en l'orientant vers les spécificités des filles anorexiques. Cependant, compte tenu de la faible prévalence de l'anorexie chez les garçons, ces dernières recommandations risquent de représenter un défi de taille pour le chercheur désirant se lancer dans un tel projet. Finalement, il serait pertinent de favoriser les études multi-sites ainsi que les études longitudinales pour nous permettre d'avoir une meilleure connaissance de l'évolution du trouble chez les garçons.

\section{Conclusion}

De manière générale, cette recension des écrits, bien qu'elle comprenne des études fort intéressantes inclut des études dont les résultats et les méthodologies sont très hétérogènes. La diversité des méthodes, des approches et des instruments de mesure utilisés permet, il va sans dire, une exploration plus large du trouble. Toutefois, elle rend difficile l'obtention de consensus sur les variables d'intérêt. Pour optimiser la recherche et générer des conclusions plus utiles ou facilement applicables en clinique, il serait nécessaire d'augmenter la rigueur de la recherche ainsi que de trouver un terrain d'entente entre les 
chercheurs quant aux variables à étudier ainsi qu'aux instruments de mesure à utiliser pour le faire. Ce faisant, il serait plus aisé de comparer les études entre elles sans devoir faire des inférences quant au propos des auteurs et il serait nettement plus facile d'avoir des consensus portant sur des nombres plus importants de participants. Il serait nécessaire que les chercheurs s'intéressant à l'anorexie masculine définissent clairement leurs concepts, ne fassent pas d'inférence, questionnent ou évaluent directement les participants sur les variables à l'étude et rendent accessibles les méthodes utilisées pour collecter leurs informations. Une suggestion pour les étapes à venir pourrait être de s'inspirer d'études effectuées auprès de filles anorexiques ou de garçons de la population générale présentant de bonnes qualités méthodologiques et de les reproduire auprès de garçons atteints d'anorexie.

\section{Bibliographie}

Aime, A. C., W M; Pepler, D; Jiang, D; Connolly, J. (2008). Developmental pathways of eating problems in adolescents. . International Journal of Eating Disorders., 41(8), 686-696.

Andersen, A. E. (1999). Eating disorders in gay males. Psychiatric Annals, 29(4), 206-212.

*Bailer, U., De Zwaan, M. \& Kasper, S. (1999). Atypical eating disorder in a mal patient. International journal of psychiatry and clinical practice, 3, 137-139.

*Bassett, J. D. (2002). Gender conflict, object representation, and body image in male anorexia: An investigation of eight subjects. Smith College Studies in Social Work, 72(3), 359-377.

*Bean, P., Loomis, C. C., Timmel, P., Hallinan, P., Moore, S., Mammel, J. \& Weltzin, T. (2004). Outcome variables for anorexic males and females one year after discharge from residential treatment. Journal of addictive diseases, 23 (2), 83-94.

Becker, A. E., Grinspoon, S. K, Klibanski, A. \& Herzog, D. B. (1999). Eating Disorders. The New England Journal of Medicine 340(14), 1092-1098.

*Bercé, C. V., A. \& Nicot, E. (2009). Quand l'anorexie n'est plus seulement féminine. Pratiques psychologiques, 15, 121-136. 
*Bonne, O., Bashi, R. \& Berry, E. (1996). Anorexia nervosa following gastroplasty in the males : two cases. International journal of eating disorders, 19 (1),105-108.

Bramon-Bosch, E. T., Nicholas A; Treasure, Janet L. (2000). Eating disorders in males: A comparison with female patients. European Eating Disorders Review., 8(4), 321-328.

Bryant-Waugh, R. L. (2007). Overview of the eating disorders. In B. E. B.-W. Lask, Rachel [Ed]. (Ed.), Eating disorders in childhood and adolescence (3rd edition ed., pp. 35-50). New York: Routledge.

Burns, T. C., A. H. (1984). Outcome of anorexia nervosa in males. British Journal of Psychiatry 145(3), 319-325.

Burns, T. C., A. H. (1985). Factor affecting pronosis in males anorexics. Journal of Psychiatric Research, 19(2/3), 323-328.

*Carlat, D. J. C., Carlos A Jr.; Herzog, David B. (1997). Eating disorders in males: A report on 135 patients. The American Journal of Psychiatry, 154(8), 1127-1132.

*Chambry, J., \& Agman, G. (2006). L'anorexie mentale masculine à l'adolescence. La Psychiatrie de l'Enfant, 49(2), 477-511.

Chambry, J., Corcos, M., Guilbaud, O., Jeammet, P. (2002). L'anorexie mentale masculine: réalités et perspectives. Annales de médecine interne, 153(3), 1S61-61S67.

*Cheung, P. \& Wilder-Smith, E. (1995). Anorexia nervosa and schizophrenia un a male chinese. International journal of eating disorders, 18(1), 103-106.

Comerci, G. D., Kilbourne, Katherine A. \& Harrisson, Gail G. (1989). Eating disorders: Obesity, Anorexia Nervosa, and Bulimia. In A. D. Hofman, Greydanus, Donald E. (Ed.), Adolescent Medecine Second Edition.

*Crisp, A. G. S., Joughin, N., McClelland, L., Rooney, B., Nielsen, S., Bowyer, C., Halek, C., Hartman, D., Tattersall, M., Hugo, P., Robinson, D., Atkinson, R. \& Clifton, A. (2006). Anorexia nervosa in males: similarities and differences to anorexia nervosain females. European eating disorders review, 14 (3), 164-167

Crosscope-Happel, C. H., David E; Getz, Hildy G; Hayes, Gerald L. (2000). Male anorexia nervosa: A new focus. Journal of Mental Health Counseling, 22(4), 365-370.

*Daini, S., Darotti, L., Manzo, A., Minerva, C. \& Mancano, G. (2008). A projective sight on male anorexia : a rorschach test exploration. Journal of projective psychology \& mental health, 15 (1), 65-72.

Domine, F. B., A; Akre, C; Michaud, P-A; Suris, J-C. . (2009). Disordered eating behaviors: What about boys? Journal of Adolescent Health., 44(2), 111-117.

* Eliot, A. O. \& Baker, C. W. (2001). Eating disordered adolescent males. Adolescence, 36 (143) 535-543.

*Fassino, S. , Giovanni, A.D; Leombruni, P.; Amianto, F.; Rovera, G.; Rovera, G. G. (2001). Temperament and character in Italian men with anorexia nervosa: A controlled study with the Temperament and Character Inventory. Journal of Nervous and Mental Disease, 189(11), 788-794. 
Fernandez-Aranda, F., Aitken, A., Badia, A., Gimenez, L., Solano, R., Collier, D., et al. (2004). Personality and Psychopathological Traits of Males with an Eating Disorder. European Eating Disorders Review, 12(6), 367-374.

Fichter, M. M., Daser, C. and Postpischil, F. (1985). Anorexic syndromes in the males. journal of Psychiatric Research, 19(2/3), 305.

*Frasciello, L. M \& Willard, S. G. (1995) Anorexia nervosa in males: a case report and review of the literature. Clinical Social Work Journal, 23 (1), 47-58.

*Frankel, G. J. \& Halmi, K. A. (2003). An adolescent with anorexia nervosa and gastrointestinal stromal tumors. The American Journal of Psychiatry, 126 (6), 10531056.

Freeman, A. C. (2005). Eating disorders in males: A review. South African Psychiatry Review, 8(2), 58-64.

Gadalla, T. M. (2008). Psychiatric comorbidity in eating disorders: comparison of men and women. Journal of Men's Health, 5(3), 209-217.

Garfinkel, P. E., \& Garner, D. M. (Eds.). (1982). Anorexia nervosa : a multidimensional perspective. New York: Brunner/Mazel.

*Gila, A. C., Josefina, C., J. \& Toro, J. (2005). Anorexia nervosa in males adolescents: body image, eating attitudes and psychological traits. Journal of Adolescent Health, 36 (3), 221-226.

Haas, H. L. C. J. R. (2001). Psychology of an eating disorder. In R.-M. J.J (Ed.), Eating disorders in women and children: prevention, stress management and treatment. (pp. 39-48). Boca Raton: CRC Press.

Halmi, K. A., Sunday, S. R., Strober, M., Kaplan, A., Woodside, D., Fichter, M., et al. (2000). Perfectionism in anorexia nervosa: Variation by clinical subtype, obsessionality, and pathological eating behavior. The American Journal of Psychiatry, 157(11), 1799-1805.

*Halperin, E. N. (1996). The role of socialization in male anorexia nervosa: a case report and review of the literature. Clinical Social Work Journal, 23 (1), 47-58.

Harvey, J. A. R., John D. (2003). Eating disorders in men: Current considerations. Journal of Clinical Psychology in Medical Settings, 10(4), 297-306.

*Hepp, U., Milos, G. \& Braun-Scharm, H. (2004). Gender identity disorders and anorexia nervosa in male monozygotic twins. International Journal of Eating Disorders, 35 (2), 239-243.

Herzog, D. B., Norman D. K., Gordon C. and Pepose M.. (1984). Sexual conflict and eating disorders in 27 males. american Journal of Psychiatry, 141(8), 989-990.

Herzog, D. B. G., Dara N; Dorer, David J; Flores, Andrea T; Ekeblad, Elizabeth R; Richards, Ana; Blais, Mark A; Keller, Martin B. (2000). Mortality in eating disorders: A descriptive study. International Journal of Eating Disorders, 28(1), 20-26. 
*Lai, K., Y. C, Pang A. H. T. \& Wong, C. K. (1995). Case study : Early onset anorexia nervosa in a chinese boy. Journal of the American Academy of Child \& Adolescent Psychiatry, 34 (3), 383-386.

Linschied, R. T.(2003). Anorexia Nervosa and Bulimia Nervosa. In M. C. Roberts (Ed.), Handbook of pediatric psychology, Third edition (pp. 636-651). New York: Guilford Press.

Lock, J., Reisel, B., \& Steiner, H. (2001). Associated health risks of adolescents with disordered eating: How different are they from their peers? Results from a high school survey. Child Psychiatry and Human Development, 31(3), 249-265. doi:

Lock, J. D. (2009). Trying to fit square pegs in round holes: Eating disorders in males. Journal of Adolescent Health., 44(2), 99-100.

*Lowenstein, L. F. (1994). Anorexia nervosa in boys : a review of the recent literature and a case treated in therapeutic community. Family therapy 21 (3),233-239.

Muise, A. M. S., Debra G; Arbess, Gordon. (2003). Eating disorders in adolescent boys: A review of the adolescent and young adult literature. Journal of Adolescent Health., 63(6), 427-435.

Olivardia, R., Pope, H. G., Mangweth, B., \& Hudson, J. I. (1995). Eating disorders in college men. The American Journal of Psychiatry, 152(9), 1279-1285.

Olivardia, R. P., Harrison G; Mangweth, Barbara; Hudson, James I. . (1995). Eating disorders in college men. The American Journal of Psychiatry, 152(9), 1279-1285.

Oyebode, F., Boodhoo, J. A. \& Schapira, K. . (1988). Anorexia nervosa in males: clinical features and outcomes. International Journal of Eating Disorders, 7(1), 121-124.

Peebles, R. (2007). Diagnosis of eating disorder impeded by gender, ethnicity. Psychiatric Annals, 37(6), 392.

Pratt, H. D., Phillips, Elaine L., Greydanus, D, E., Patel, Dilip R. (2003). Eating Disorders in the adolescent population: future directions. Journal of adolescent research, 18(3), 297-317.

Ray, S. L. (2004). Eating Disorders in Adolescent Males. Professional School Counseling, $8(1), 98-10$.

Reijonen, J. H., Pratt, H. D., Patel, D.R, \& Greydanus, D.E. (2003). Eating disorders in the adolescent population: an overview. Journal of adolescent research 18(3), 209-222. Robert-McComb, J. J. (2001). Eating disorders. In J. J. Robert-McComb (Ed.), Eating Disorders in Women and Children: Prevention, Stress Management and Treatment (pp. 3-37). Boca Raton: CRC Press.

Romeo, F. (1994). Adolescent boys and anorexia nervosa. Adolescence, 29(115), 643-647.

*Sharp, C. W., Clark, S. A., Dunan, J. R., Blackwood, D. H. \& Shapiro, C. M. (1994). Clinical presentation of anorexia nervosa in malesL 24 new cases. International Journal of Eating Disorders, 15 (2), 125-134. 
Steinhausen, H.-C. (2002). The outcome of anorexia nervosa in the 20th century. The American Journal of Psychiatry, 159(8), 1284-1293.

Stice, E., \& Shaw, H. E. (2002). Role of body dissatisfaction in the onset and maintenance of eating pathology: A synthesis of research findings. Journal of Psychosomatic Research, 53(5), 985-993.

Striegel-Moore, R. H. (1997). Risk factors for eating disorders. Jacobson, Marc S [Ed], 98109.

Striegel-Moore, R. H., \& Bulik, C. M. (2007). Risk factors for eating disorders. American Psychologist, 62(3), 181-198.

*Tong, J., Miao, S. J., Wang, J. Zhang, J. J., Wu H. M., Li, T. \& Hsu, L. K. G. (2005). Five cases of male eating disorders in central china. International Journal of Eating Disorders, 37 (1), 72-75.

*Toro, J, Castro, J., Gila, A. \& Pombo, C. (2005). Assessment of sociocultural influences on the body shap model in adolescent males with anorexia nervosa. European Eating Disorders Review, 13, 351-359.

Walcott, D. D., Pratt, Helen D., Patel, Dilip R. (2003). Adolescent and eating disorders: gender, racial, ethnic, socioculturalm, and socioeconomic issues. Journal of adolescent research, 183(3), 223-243.

*Walters, E. \& Whitehead, L. (1997). Anorexia nervosa in a young boy with gender identity disorder of childhood: a case report. Clinical Child Psychology and Psychiatry, 3 (2), 463-467.

Weltzin, T. E. W., Nicolette; Franczyk, David; Burnett, Kevin; Klitz, Christine; Bean, Pamela. (2005). Eating disorders in men: Update. Journal of Men's Health \& Gender, 2(2), 186-193.

Woodside, D., Bulik, C. M., Thornton, L., Klump, K. L., Tozzi, F., Fichter, M. M., et al. (2004). Personality in men with eating disorders. Journal of Psychosomatic Research, 57(3), 273-278.

Woodside, D., Garfinkel, P. E., Lin, E., Goering, P., \& Kaplan, A. S. (2001). Comparisons of men with full or partial eating disorders, men without eating disorders, and women with eating disorders in the community. The American Journal of Psychiatry, 158(4), 570-574.

*Young, S. (1998). Body talk: some thoughts on anorexia. Australian and New Zealand Journal of Family Therapy, 19 (2), 63-67. 


\section{Annexe 1}

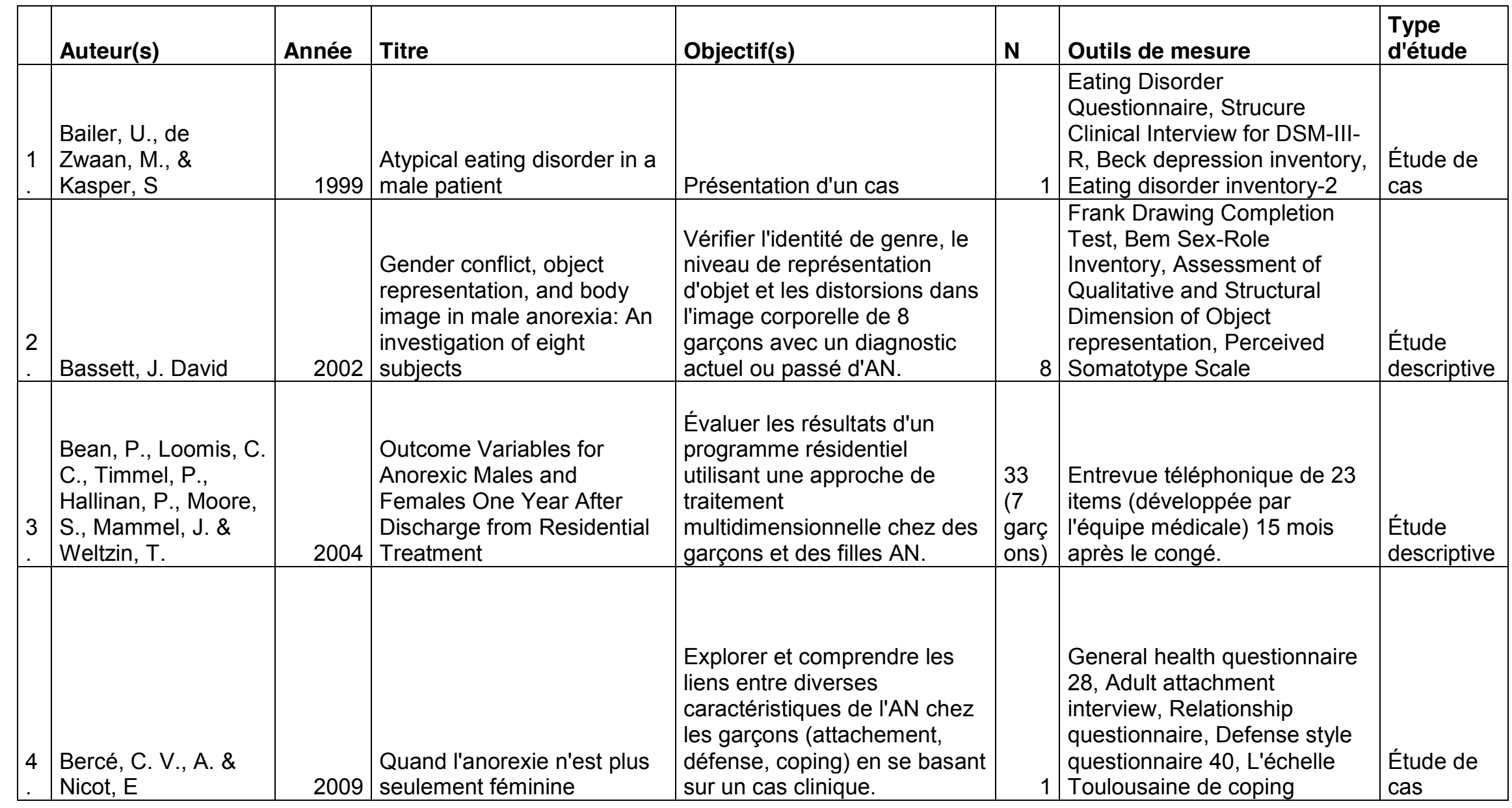




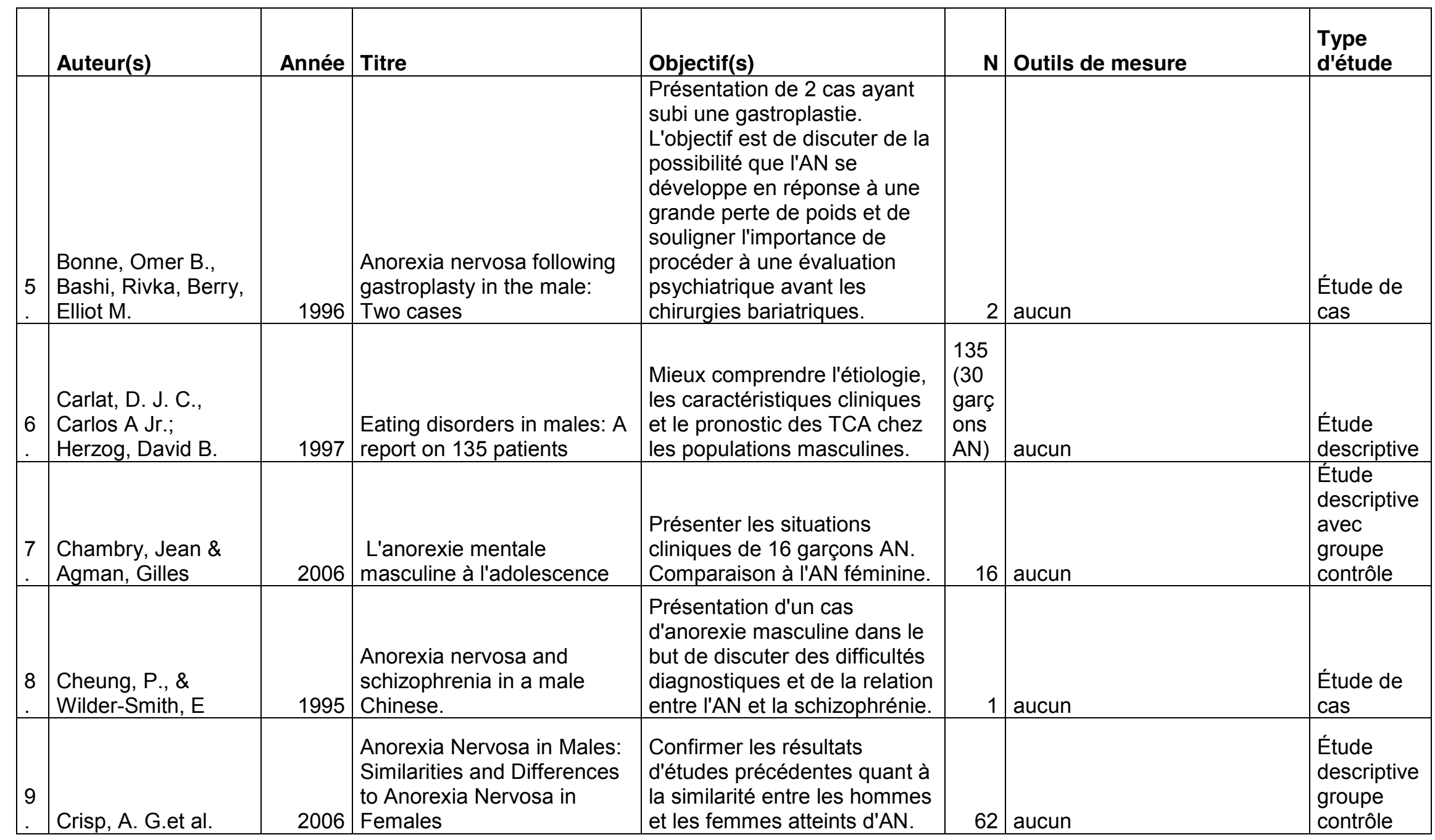




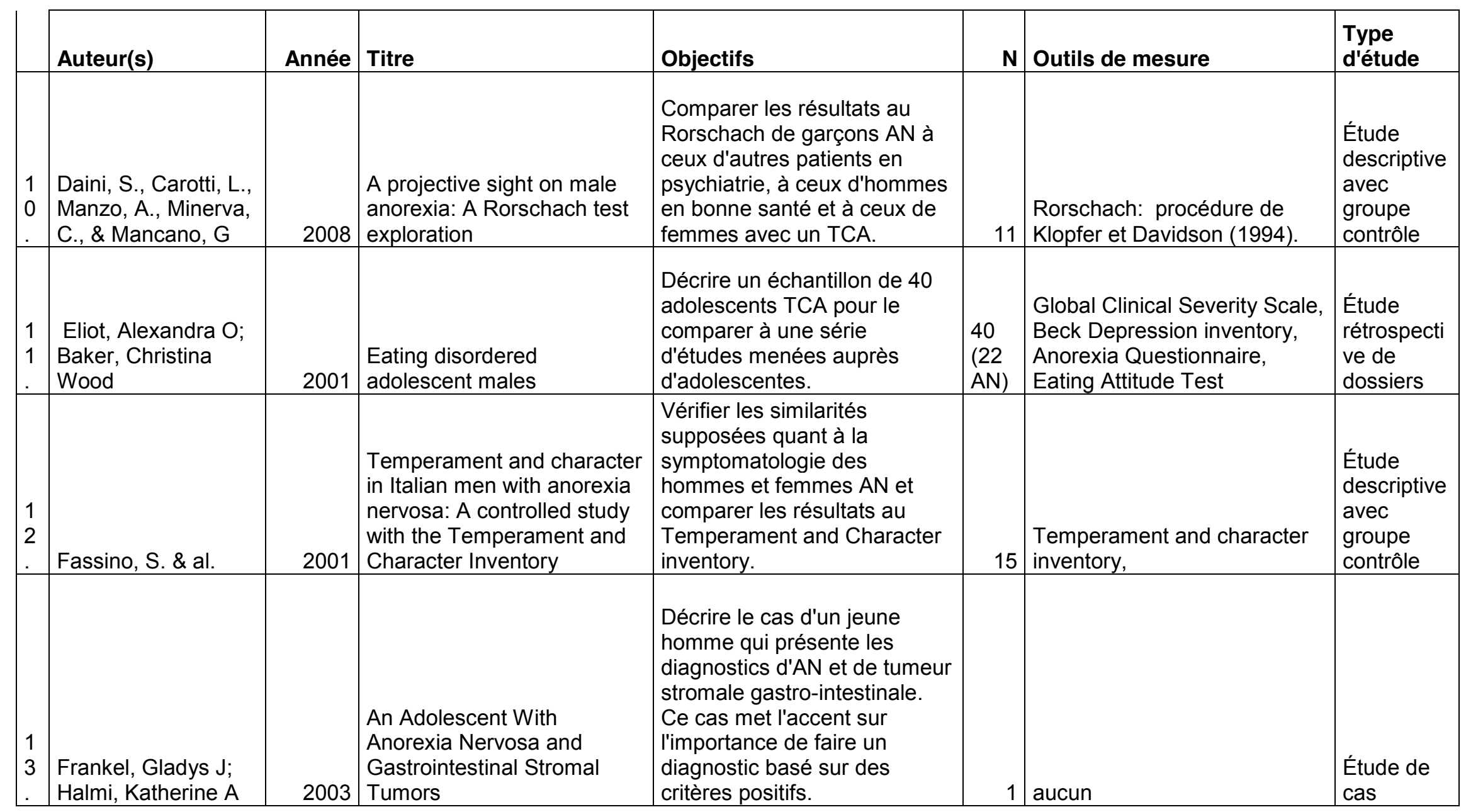




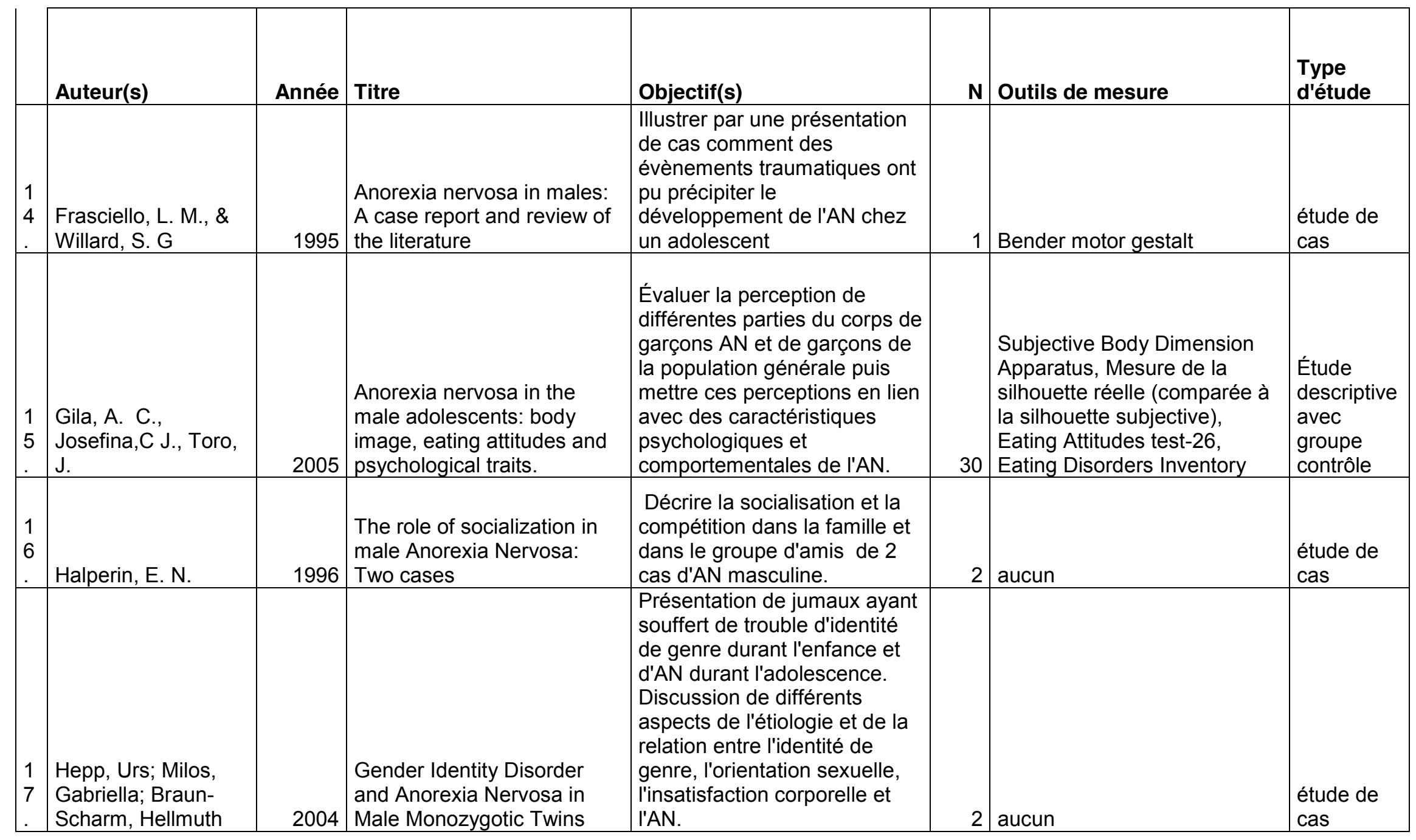




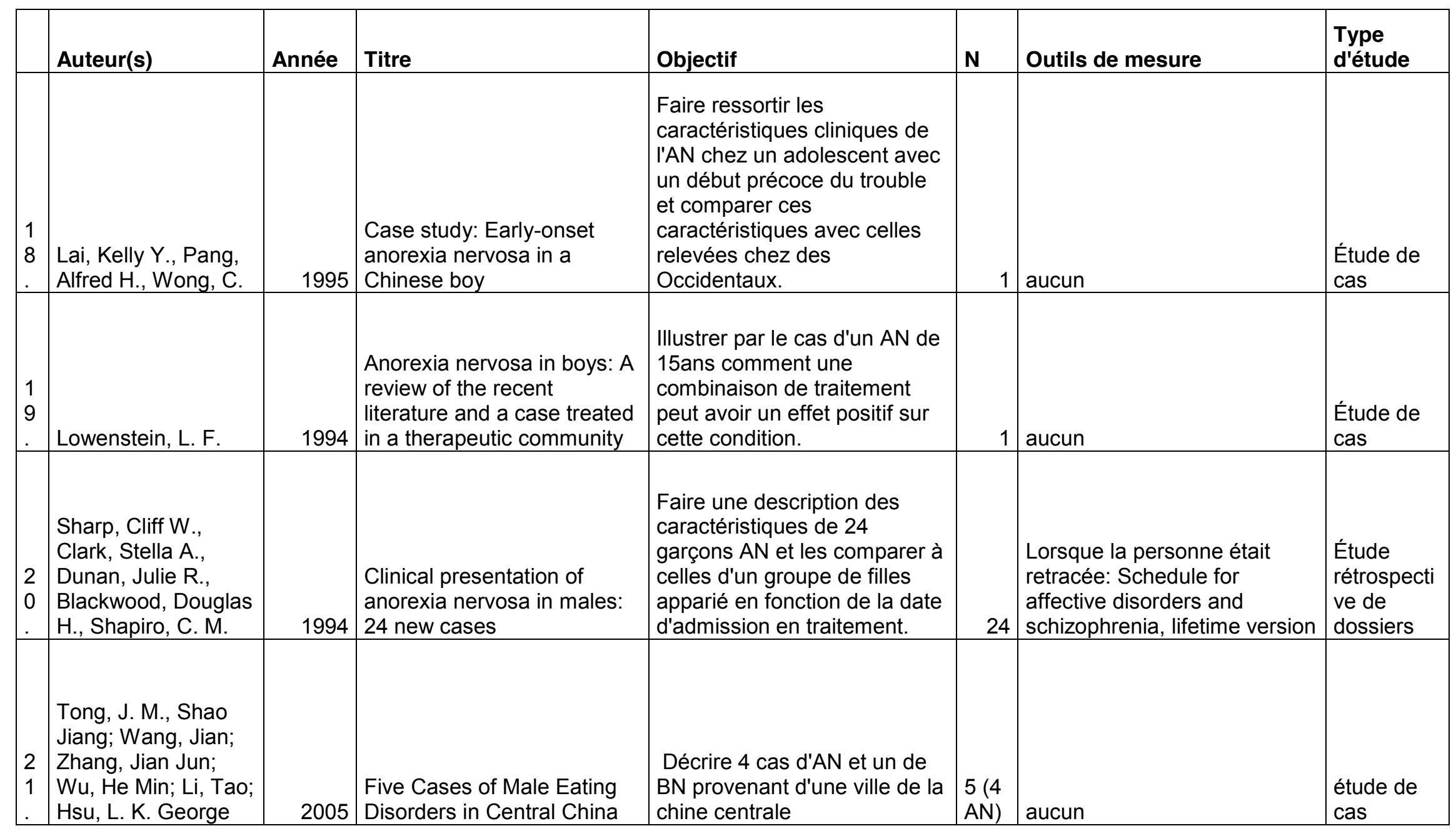




\begin{tabular}{|c|c|c|c|c|c|c|c|}
\hline & Auteur(s) & Année & Titre & Objectif(s) & $\mathbf{N}$ & Outils de mesure & $\begin{array}{l}\text { Type } \\
\text { d'étude }\end{array}$ \\
\hline $\begin{array}{l}2 \\
2\end{array}$ & $\begin{array}{l}\text { Toro, J. C., J; Gila, } \\
\text { A; Pombo, C. }\end{array}$ & 2005 & $\begin{array}{l}\text { Assessment of sociocultural } \\
\text { influences on the body } \\
\text { shape model in adolescent } \\
\text { males with anorexia nervosa }\end{array}$ & $\begin{array}{l}\text { Produire un questionnaire } \\
\text { pour évaluer l'influence } \\
\text { socioculturelle sur l'idéal } \\
\text { corporel des garçons, étudier } \\
\text { le rôle des influences } \\
\text { socioculturelles dans le } \\
\text { développement de l'AN chez } \\
\text { les garçons et comparer les } \\
\text { résultats à ceux obtenus } \\
\text { auprès de populations } \\
\text { féminines. }\end{array}$ & 34 & $\begin{array}{l}\text { CIMEC (Questionnaire sur les } \\
\text { influences sur l'idéal corporel } \\
\text { - body shape model-), EAT- } \\
\text { 26, Mesures } \\
\text { anthropométriques }\end{array}$ & $\begin{array}{l}\text { Étude } \\
\text { descriptive } \\
\text { avec } \\
\text { groupe } \\
\text { contrôle }\end{array}$ \\
\hline $\begin{array}{l}2 \\
3\end{array}$ & $\begin{array}{l}\text { Walters, Elizabeth; } \\
\text { Whitehead, Linda }\end{array}$ & 1995 & $\begin{array}{l}\text { Anorexia nervosa in a young } \\
\text { boy with gender identity } \\
\text { disorder of childhood: A } \\
\text { case report }\end{array}$ & $\begin{array}{l}\text { Description d'un cas de } 12 \\
\text { ans présentant une AN et un } \\
\text { trouble d'identité de genre et } \\
\text { discussion sur leurs étiologies } \\
\text { et sur la présence de } \\
\text { problématiques } \\
\text { psychosexuelles dans les } \\
\text { TCA. }\end{array}$ & 1 & aucune & $\begin{array}{l}\text { Étude de } \\
\text { cas }\end{array}$ \\
\hline
\end{tabular}

